TRANSACTIONS OF THE

AMERICAN MATHEMATICAL SOCIETY

Volume 351, Number 10, Pages 4043-4067

S 0002-9947(99)02206-0

Article electronically published on April 27, 1999

\title{
ALEXANDER INVARIANTS OF COMPLEX HYPERPLANE ARRANGEMENTS
}

\author{
DANIEL C. COHEN AND ALEXANDER I. SUCIU
}

\begin{abstract}
Let $\mathcal{A}$ be an arrangement of $n$ complex hyperplanes. The fundamental group of the complement of $\mathcal{A}$ is determined by a braid monodromy homomorphism, $\alpha: F_{s} \rightarrow P_{n}$. Using the Gassner representation of the pure braid group, we find an explicit presentation for the Alexander invariant of $\mathcal{A}$. From this presentation, we obtain combinatorial lower bounds for the ranks of the Chen groups of $\mathcal{A}$. We also provide a combinatorial criterion for when these lower bounds are attained.
\end{abstract}

\section{INTRODUCTION}

Let $\mathcal{A}=\left\{H_{1}, \ldots, H_{n}\right\}$ be an arrangement of hyperplanes in $\mathbb{C}^{d}$, with complement $M=\mathbb{C}^{d} \backslash \bigcup_{i=1}^{n} H_{i}$, and group $G=\pi_{1}(M)$. Let $M^{\prime}$ be the maximal abelian cover, corresponding to the abelianization ab $: G \rightarrow \mathbb{Z}^{n}$. The action of $\mathbb{Z}^{n}$ on $M^{\prime}$ puts on $H_{*}\left(M^{\prime}\right)$ the structure of a module over the group ring $\mathbb{Z} \mathbb{Z}^{n}$. This ring can be identified with the ring of Laurent polynomials $\Lambda=\mathbb{Z}\left[t_{1}^{ \pm 1}, \ldots, t_{n}^{ \pm 1}\right]$, with $t_{i}$ corresponding to a standardly oriented meridional loop around $H_{i}$. The object of our study is the Alexander invariant, $B(\mathcal{A})=H_{1}\left(M^{\prime}\right)$, viewed as a module over the ring $\Lambda$.

Let $L(\mathcal{A})$ denote the intersection lattice of $\mathcal{A}$, with rank function given by codimension (see Orlik and Terao [27] as a general reference for arrangements). Let $s$ denote the cardinality of $L_{2}(\mathcal{A})$, the set of rank two elements in $L(\mathcal{A})$. From the defining polynomial of $\mathcal{A}$, one can compute the Moishezon-Libgober braid monodromy homomorphism, $\alpha: F_{s} \rightarrow P_{n}$, see [7]. This homomorphism determines a finite presentation for the group of the arrangement: $G=\left\langle t_{1}, \ldots, t_{n} \mid \alpha_{k}\left(t_{i}\right)=t_{i}\right\rangle$, where $\alpha_{1}, \ldots, \alpha_{s}$ generate the image of $\alpha$. The braid monodromy may also be used to obtain a finite presentation for the Alexander invariant $B(\mathcal{A})$. We accomplish this here, by means of the Gassner representation, $\Theta: P_{n} \rightarrow \operatorname{GL}(n, \Lambda)$, the Fox calculus, and homological algebra.

Surprisingly, the size of the presentation depends only on the first two betti numbers of the complement: there are $\left(\begin{array}{l}n \\ 2\end{array}\right)$ generators and $\left(\begin{array}{l}n \\ 3\end{array}\right)+b_{2}(M)$ relations.

Received by the editors March 24, 1997 and, in revised form, September 9, 1997.

1991 Mathematics Subject Classification. Primary 14H30, 52B30, 57M05; Secondary 20F14, 20F36

Key words and phrases. Arrangement, braid monodromy, Alexander invariant, Chen groups.

The first author was partially supported by grant LEQSF(1996-99)-RD-A-04 from the Louisiana Board of Regents and by a grant from the Louisiana State University Council on Research.

The second author was partially supported by N.S.F. grant DMS-9504833, and an RSDF grant from Northeastern University.

(C)1999 American Mathematical Society 
When $\mathcal{A}$ is the complexification of a real arrangement, the presentation of $B(\mathcal{A})$ can be simplified to $\left(\begin{array}{l}n \\ 2\end{array}\right)-b_{2}(M)$ generators and $\left(\begin{array}{l}n \\ 3\end{array}\right)$ relations. More generally, if $G$ is the group of a collection of $s$ basis-conjugating automorphisms of a finitely generated free group $F_{n}$, our methods yield a presentation of $B(G)$ with $\left(\begin{array}{l}n \\ 2\end{array}\right)$ generators and $\left(\begin{array}{l}n \\ 3\end{array}\right)+n s$ relations. In particular, the Alexander invariant of any pure link has such a presentation (with $s=1$ ). This should be compared with the general situation for links in $S^{3}$, where there is no upper bound on the number of relations, see Traldi [31].

Note that the Alexander invariant is isomorphic to $G^{\prime} / G^{\prime \prime}$ (with the usual $G / G^{\prime}$ action), and so depends only on the isomorphism type of $G$. Consequently, we may obtain invariants of an arrangement $\mathcal{A}$ from the module $B=B(\mathcal{A})$ and its presentation. For instance, if $\Delta$ is a presentation matrix for $B$, the elementary ideal $E_{k}(B)$ is defined to be the ideal generated by the codimension $k$ minors of $\Delta$. It is well-known that these ideals depend only on the module $B$. These ideals, and the closely related characteristic varieties, arise in the study of plane algebraic curves; see for instance the recent works of Hironaka [20] and Libgober [22]. The structure of the elementary ideals and characteristic varieties of the Alexander invariant of an arrangement will be the subject of a future work.

In this paper, we focus on Chen groups. The Chen groups of $G$ are the lower central series quotients of the maximal metabelian quotient $G / G^{\prime \prime}$. Using an observation of Massey [23] relating the Chen groups and the Alexander invariant, together with Mora's tangent cone algorithm, we obtain an algorithm for computing the Chen groups of an arrangement from the presentation of the Alexander invariant $B$. The ranks of the Chen groups often serve to distinguish the groups of combinatorially "similar" arrangements. This is particularly useful for fiber-type arrangements, where the ranks of the lower central series quotients of $G$ itself are determined by the exponents of the arrangement. On the other hand, we know of no combinatorially equivalent arrangements whose Chen groups differ.

The precise relation between the Chen groups and the intersection lattice of a central arrangement $\mathcal{A}$ is not known. We obtain partial results toward this end here. To each element $V \in L_{2}(\mathcal{A})$, we associate a "local" Alexander invariant $B_{V}$. Algebraic considerations yield a surjective homomorphism $B \rightarrow B^{\text {cc }}$, where $B^{\mathrm{cc}}=\bigoplus_{V} B_{V}$ is the "coarse combinatorial Alexander invariant" of $\mathcal{A}$, determined by (only) the multiplicities of the elements of $L_{2}(\mathcal{A})$. From this map, we obtain combinatorial lower bounds on the ranks of the Chen groups of $\mathcal{A}$. These ranks are determined by the $I$-adic completion, $\widehat{B}$, of the Alexander invariant $B$, where $I$ is the augmentation ideal of $\Lambda$. We find a combinatorial criterion for when the completion of the Alexander invariant of $\mathcal{A}$ decomposes as a direct sum, i.e., $\widehat{B} \stackrel{\sim}{\longrightarrow}$ $\widehat{B}^{\mathrm{cc}}$. We also obtain a combinatorial formula for the rank of the third Chen group of any arrangement. The above results may be viewed as evidence that the ranks, $\theta_{k}$, of the Chen groups of $\mathcal{A}$ are combinatorially determined. In [5], we conjectured an explicit combinatorial formula for $\theta_{k}$, for sufficiently large $k$. This formula involved the number $\beta$ of subarrangements of $\mathcal{A}$ lattice-isomorphic to the braid arrangement $\mathcal{A}_{4} \subset \mathbb{C}^{4}$. In the present context, we show by example that if $\beta \geq 1$, then the map $\widehat{B} \rightarrow \widehat{B}^{\text {cc }}$ is not an isomorphism. Other examples exhibit combinatorially different ways this map can fail to be an isomorphism. These provide counterexamples to the aforementioned formula, and illustrate the subtlety of the relationship between the Chen groups and the lattice of an arrangement. 
Our results on Chen groups parallel a portion of Falk's work on the LCS quotients of an arrangement group. The combinatorial lower bounds we obtain for the ranks of the Chen groups are analogous to those for the ranks, $\phi_{k}$, of the LCS quotients found in [13]. Moreover, the formula we obtain for $\theta_{3}=\phi_{3}$ may be viewed as dual to the description of $\phi_{3}$ found in [12], [13]. The precise relationship between the Chen groups and LCS quotients of an arrangement will be explored elsewhere.

The structure of the paper is as follows:

- In section 1, we review Alexander invariants and Chen groups, and present a Groebner basis algorithm for determining the latter. The section concludes with an analysis of the Alexander invariant and Chen groups of a product of spaces.

- In section 2, we introduce our basic computational tools: the Fox free differential calculus and the Magnus representations.

- In section 3, we study the Alexander invariant of the group of a free automorphism. An explicit presentation is given when the automorphism is basis-conjugating.

- In section 4, we find presentations for the local Alexander invariants of an arrangement.

- In section 5, the presentation for the Alexander invariant of an arrangement is obtained.

- In section 6 , the homomorphism $B \rightarrow B^{\mathrm{cc}}$ is defined, and its completion proven to be an isomorphism when a certain criterion is satisfied.

- In section 7, the aforementioned criterion is shown to be combinatorial, and lower bounds on the ranks of the Chen groups of an arrangement are obtained.

- In section 8 , we illustrate our results by means of several explicit examples.

Conventions. Given a group $G$, we will denote by $\operatorname{Aut}(G)$ the group of right automorphisms of $G$, with multiplication $\alpha \cdot \beta=\beta \circ \alpha$. We will regard all modules over the group ring $\mathbb{Z} G$ as left modules. Elements of the free module $(\mathbb{Z} G)^{n}$ are viewed as row vectors, and $\mathbb{Z} G$-linear maps $(\mathbb{Z} G)^{n} \rightarrow(\mathbb{Z} G)^{m}$ are viewed as $n \times m$ matrices which act on the right (so that the matrix of $B \circ A$ is $A \cdot B$ ). We will write $A^{\top}$ for the transpose of $A$, and $\left(A_{1} \cdots A_{s}\right)^{\top}$ for $\left(\begin{array}{l}A_{1} \\ \dddot{A}_{s}\end{array}\right)$. If $\phi: G \rightarrow H$ is a homomorphism, $\tilde{\phi}: \mathbb{Z} G \rightarrow \mathbb{Z} H$ denotes its $\mathbb{Z}$-linear extension to group rings. We will abuse notation and also write $\tilde{\phi}:(\mathbb{Z} G)^{n} \rightarrow(\mathbb{Z} H)^{n}$ for the map $\bigoplus_{1}^{n} \tilde{\phi}$.

\section{Alexander invariants, Chen groups, and products}

We start by reviewing the definition of the Alexander invariant of a finite complex. We then present an algorithm for computing the ranks of the Chen groups of a group, based on a presentation of this module. Finally, we determine the structure of the Alexander invariant of a product of spaces in terms of those of the factors.

1.1. Alexander Invariants. Let $M$ be a path-connected space that has the homotopy type of a finite $\mathrm{CW}$-complex. Let $G=\pi_{1}(M, *)$ be the fundamental group, and $K=H_{1}(M)$ its abelianization. Let $p: M^{\prime} \rightarrow M$ be the maximal abelian cover. The action of $K$ on $M^{\prime}$ passes to an action of $K$ on the homology groups $H_{*}\left(M^{\prime}\right)$. This defines on $H_{*}\left(M^{\prime}\right)$ the structure of a module over the group ring $\mathbb{Z} K$. The $\mathbb{Z} K$-module $B=H_{1}\left(M^{\prime}\right)$ is called the (first) Alexander invariant of $M$. Closely related to it is the (first) Alexander module, $A=H_{1}\left(M^{\prime}, p^{-1}(*)\right)$. These 
two modules, together with the augmentation ideal $I=I K=\operatorname{ker}(\epsilon: \mathbb{Z} K \rightarrow \mathbb{Z})$, comprise the Crowell exact sequence, $0 \rightarrow B \rightarrow A \rightarrow I \rightarrow 0$, of [10].

The two Alexander modules depend only on the group $G$. Indeed, $A=\mathbb{Z} K \otimes_{\mathbb{Z} G}$ $I G$, with $K=G / G^{\prime}$ acting by multiplication on the left factor, and $B=G^{\prime} / G^{\prime \prime}$, with the action of $K$ defined by the extension $1 \rightarrow G^{\prime} / G^{\prime \prime} \rightarrow G / G^{\prime \prime} \rightarrow G / G^{\prime} \rightarrow 1$. Since $M$ is by assumption a finite complex, $G$ is a finitely presented group. Hence, the $\mathbb{Z} K$-module $A$ is finitely presented; Fox's free differential calculus provides an explicit presentation (see [11], and also sections 2 and 3). Less evident, but still true, is the fact that $B$ also admits a finite presentation as a $\mathbb{Z} K$-module (see [10], [23], and also section 3).

1.2. Chen Groups. Let $\Gamma_{k}(G)$ denote the $k^{\text {th }}$ lower central series subgroup of $G$, defined inductively by $\Gamma_{1}(G)=G$ and $\Gamma_{k+1}(G)=\left[\Gamma_{k}(G), G\right]$ for $k \geq 1$. The projection of $G$ onto its maximal metabelian quotient $G / G^{\prime \prime}$ induces an epimorphism

$$
\frac{\Gamma_{k}(G)}{\Gamma_{k+1}(G)} \rightarrow \frac{\Gamma_{k}\left(G / G^{\prime \prime}\right)}{\Gamma_{k+1}\left(G / G^{\prime \prime}\right)}
$$

from the $k^{\text {th }}$ lower central series quotient of $G$ to the $k^{\text {th }}$ Chen group of $G$. Since $G$ is finitely presented, these quotients are finitely generated abelian groups, whose ranks we will denote by $\phi_{k}$, respectively $\theta_{k}$. It is readily seen that $\phi_{k}=\theta_{k}$ for $k \leq 3$, and $\phi_{k} \geq \theta_{k}$ for $k>3$.

The Chen groups of $G$ can be determined from the Alexander invariant of $G$. Indeed, Massey [23] noted the following isomorphism, for $k \geq 2$ :

$$
\frac{\Gamma_{k}\left(G / G^{\prime \prime}\right)}{\Gamma_{k+1}\left(G / G^{\prime \prime}\right)}=\frac{I^{k-2} B}{I^{k-1} B}
$$

Thus, the Chen groups are determined by gr $B=\bigoplus_{k \geq 0} I^{k} B / I^{k+1} B$, viewed as a graded module over the graded ring gr $\mathbb{Z} K=\bigoplus_{k \geq 0} I^{k} / I^{k+1}$.

Now assume $K$ is free abelian, and fix a system of generators, $t_{1}, \ldots, t_{n}$. The group ring $\mathbb{Z} K$ can be identified with the ring of Laurent polynomials in $n$ variables, $\Lambda=\mathbb{Z}\left[t_{1}^{ \pm 1}, \ldots, t_{n}^{ \pm 1}\right]$. The ring $\Lambda$ can be viewed as a subring of the formal power series ring $P=\mathbb{Z}\left[\left[x_{1}, \ldots, x_{n}\right]\right]$ via the "Magnus embedding," given by $t_{i} \mapsto 1-x_{i}$ and $t_{i}^{-1} \mapsto \sum_{k=0}^{\infty} x_{i}^{k}$. Let $\widehat{\Lambda}=\lim _{\longleftarrow} \Lambda / I^{k}$ be the completion of $\Lambda$ relative to the $I$-adic topology. Then, the Magnus embedding extends to a ring isomorphism $\widehat{\Lambda} \stackrel{\sim}{\longrightarrow} P$.

Consider the $\mathfrak{m}$-adic filtration on $P$, where $\mathfrak{m}=\left\langle x_{1}, \ldots, x_{n}\right\rangle$, and its associated graded ring, gr $P=\bigoplus_{k \geq 0} \mathfrak{m}^{k} / \mathfrak{m}^{k+1}$. As is well-known, this ring is isomorphic to the polynomial ring $R=\mathbb{Z}\left[x_{1}, \ldots, x_{n}\right]$. Moreover, the Magnus embedding induces a graded ring isomorphism gr $\Lambda \stackrel{\sim}{\longrightarrow}$ gr $P=R$. Let $\widehat{B}$ be the $I$-adic completion of $B$, and gr $\widehat{B}=\bigoplus_{k \geq 0} \mathfrak{m}^{k} \widehat{B} / \mathfrak{m}^{k+1} \widehat{B}$ the associated graded module. Then, the canonical map $B \rightarrow \widehat{B}$ induces an isomorphism gr $B \stackrel{\sim}{\longrightarrow}$ gr $\widehat{B}$ of graded modules over the ring $R$.

Combining these facts, we can restate Massey's result as follows:

Theorem $1.3([23])$. The generating series for the ranks of the Chen groups of $G$, $\sum_{k=0}^{\infty} \theta_{k+2} t^{k}$, is equal to the Hilbert series of the graded module associated to the I-adic completion of $B(G), \sum_{k=0}^{\infty} \operatorname{rank}\left(\mathfrak{m}^{k} \widehat{B} / \mathfrak{m}^{k+1} \widehat{B}\right) t^{k}$.

An immediate consequence of this theorem is that, for $k$ sufficiently large, $\theta_{k}$ is given by a polynomial in $k$. Indeed, this is just the Hilbert-Serre polynomial of gr $\widehat{B}$, see [32]. 
1.4. Groebner Bases. Let $\Lambda^{a} \stackrel{\Delta}{\longrightarrow} \Lambda^{b} \rightarrow B \rightarrow 0$ be a (finite) presentation of the Alexander invariant. Note that, by replacing the generators of the free module $\Lambda^{a}$ by suitable multiples if necessary, we may assume that the entries of the matrix of $\Delta$ are polynomials in the variables $t_{i}$. Let $J=\operatorname{im} \Delta$. A presentation for the $I$-adic completion of $B$ is given by $\widehat{\Lambda}^{a} \stackrel{\widehat{\Delta}}{\longrightarrow} \widehat{\Lambda}^{b} \rightarrow \widehat{B} \rightarrow 0$, where $\widehat{\Delta}$ is obtained from $\Delta$ via the Magnus embedding. Clearly, im $\widehat{\Delta}=\widehat{J}$. Since all the entries of the matrix for $\widehat{\Delta}$ belong to the subring $R \subset P$, we may restrict $\widehat{\Delta}$ to a map $\Delta: R^{a} \rightarrow R^{b}$, whose image, $\widehat{J} \cap R^{b}$, we denote by $\mathbf{J}$.

We must find a presentation for the associated graded module $\operatorname{gr} \widehat{B}=\operatorname{gr}\left(P^{b} / \widehat{J}\right)$. This module is isomorphic to $R^{b} / \operatorname{LT}(\mathbf{J})$, where $\operatorname{LT}(\mathbf{J})$ is the submodule of $R^{b}$ consisting of lowest degree homogeneous forms of elements in $\mathbf{J}$, see [32]. We are left with finding a finite generating set for $\operatorname{LT}(\mathbf{J})$. Such a set is provided by Mora's algorithm for obtaining the tangent cone of an affine variety at the origin, see [9], [2]. Essentially, we must determine a (minimal) Groebner basis $\mathcal{G}=\left\{g_{1}, \ldots, g_{c}\right\}$ for the module $\mathbf{J}$, with respect to a suitable monomial ordering. Then, $\operatorname{LT}(\mathbf{J})$ has Groebner basis $\operatorname{LT}(\mathcal{G})=\left\{\mathrm{LT} g_{1}, \ldots, \mathrm{LT} g_{c}\right\}$, from which we can extract a minimal Groebner basis $\mathcal{H}=\left\{h_{1}, \ldots, h_{d}\right\}$. Putting all these facts together, we obtain the following.

Theorem 1.5. The module gr $\widehat{B}$ has presentation $R^{d} \stackrel{\operatorname{gr} \widehat{\Delta}}{\longrightarrow} R^{b} \rightarrow \operatorname{gr} \widehat{B} \rightarrow 0$, where the rows of gr $\widehat{\Delta}$ constitute a minimal Groebner basis for the module generated by the rows of the matrix $\widehat{\Delta}$, obtained from a presentation matrix $\Delta$ for $B$ by replacing $t_{i}$ by $1-x_{i}$.

Example 1.6. Let $G=F_{n}$ be a free group of rank $n$. A presentation for the Alexander invariant $B$ of $G$ is given by $\Lambda^{a} \stackrel{d_{3}}{\longrightarrow} \Lambda^{b} \rightarrow B \rightarrow 0$, where $a=\left(\begin{array}{l}n \\ 3\end{array}\right), b=\left(\begin{array}{l}n \\ 2\end{array}\right)$, and $d_{3}$ is the differential in the standard $\Lambda$-resolution of $\mathbb{Z}$. In this instance, it is readily checked that the rows of the matrix $\hat{d}_{3}$ form a Groebner basis for the module J. A standard argument then yields the ranks of the Chen groups of $F_{n}: \theta_{1}=n$ and $\theta_{k}=(k-1)\left(\begin{array}{c}k+n-2 \\ k\end{array}\right)$ for $k \geq 2$, a calculation originally due to Murasugi [26] (see also [24]).

1.7. Products. Let $M_{1}$ and $M_{2}$ be two path connected finite CW-complexes, with $K_{i}=H_{1}\left(M_{i}\right)$ free abelian, and let $M_{i}^{\prime}$ be the corresponding maximal abelian covers. Then $M=M_{1} \times M_{2}$ has maximal abelian cover $M^{\prime}=M_{1}^{\prime} \times M_{2}^{\prime}$, corresponding to $K=H_{1}(M)=K_{1} \times K_{2}$.

Proposition 1.8. There is an isomorphism of $\mathbb{Z} K$-modules,

$$
H_{1}\left(M^{\prime}\right) \cong\left(\left(H_{1}\left(M_{1}^{\prime}\right) \otimes_{\mathbb{Z} K_{1}} \mathbb{Z} K\right) \otimes_{\mathbb{Z} K_{2}} \mathbb{Z}\right) \oplus\left(\left(H_{1}\left(M_{2}^{\prime}\right) \otimes_{\mathbb{Z} K_{2}} \mathbb{Z} K\right) \otimes_{\mathbb{Z} K_{1}} \mathbb{Z}\right) .
$$

Proof. By the (homological) Künneth formula, the group $H_{1}\left(M^{\prime}\right)$ is isomorphic to $H_{1}\left(M_{1}^{\prime}\right) \otimes H_{0}\left(M_{2}^{\prime}\right) \oplus H_{0}\left(M_{1}^{\prime}\right) \otimes H_{1}\left(M_{2}^{\prime}\right)$. When viewed as a $\mathbb{Z} K$-module, the first summand is isomorphic to

$$
\begin{aligned}
& \left(\left(H_{1}\left(M_{1}^{\prime}\right) \otimes_{\mathbb{Z} K_{1}} \mathbb{Z} K\right) \otimes_{\mathbb{Z} K} \mathbb{Z} K_{1}\right) \otimes\left(\left(\mathbb{Z} \otimes_{\mathbb{Z} K_{2}} \mathbb{Z} K\right) \otimes_{\mathbb{Z} K} \mathbb{Z} K_{2}\right) \\
& \quad=\left(H_{1}\left(M_{1}^{\prime}\right) \otimes_{\mathbb{Z} K_{1}} \mathbb{Z} K\right) \otimes_{\mathbb{Z} K}\left(\mathbb{Z} K_{1} \otimes \mathbb{Z} K_{2}\right) \otimes_{\mathbb{Z} K}\left(\mathbb{Z} \otimes_{\mathbb{Z} K_{2}} \mathbb{Z} K\right) \\
& \quad=\left(H_{1}\left(M_{1}^{\prime}\right) \otimes_{\mathbb{Z} K_{1}} \mathbb{Z} K\right) \otimes_{\mathbb{Z} K_{2}} \mathbb{Z},
\end{aligned}
$$

where we made use of the obvious isomorphism $\left(\mathbb{Z} K_{1} \otimes \mathbb{Z} K_{2}\right) \cong \mathbb{Z}\left(K_{1} \times K_{2}\right)$, and viewed the induced module $H_{1}\left(M_{1}^{\prime}\right) \otimes_{\mathbb{Z} K_{1}} \mathbb{Z} K$ as a $\mathbb{Z} K_{2}$-module by restriction of scalars. The second summand is treated exactly the same way. 
We want to find now a presentation for the Alexander invariant $B(M)=H_{1}\left(M^{\prime}\right)$, given presentations for the Alexander invariants $B\left(M_{i}\right)=H_{1}\left(M_{i}^{\prime}\right)$. Fix generators $t_{1}^{(i)}, \ldots, t_{n_{i}}^{(i)}$ for $K_{i}$, and use them to identify $\mathbb{Z} K_{i}$ with $\Lambda_{i}$.

Theorem 1.9. If the Alexander invariants of $M_{1}$ and $M_{2}$ have presentations

$$
\Lambda_{i}^{a_{i}} \stackrel{\Delta_{i}}{\longrightarrow} \Lambda_{i}^{b_{i}} \rightarrow B\left(M_{i}\right) \rightarrow 0,
$$

then the Alexander invariant of $M=M_{1} \times M_{2}$ has presentation

$$
\Lambda^{a} \stackrel{\left(\begin{array}{c}
\Delta_{1} \\
D_{2}^{b_{1}}
\end{array}\right) \oplus\left(\begin{array}{c}
\Delta_{2} \\
D_{1}^{b_{2}}
\end{array}\right)}{\longrightarrow} \Lambda^{b} \rightarrow B(M) \rightarrow 0
$$

where $a=a_{1}+n_{2} b_{1}+a_{2}+n_{1} b_{2}, b=b_{1}+b_{2}$, and $D_{i}=\left(t_{1}^{(i)}-1, \ldots, t_{n_{i}}^{(i)}-1\right)^{\top}$.

Proof. Let us look at the first summand in the direct sum decomposition of $H_{1}\left(M^{\prime}\right)$ from Proposition 1.8. It is the tensor product over $\mathbb{Z} K$ of two induced modules. The first one is the $\mathbb{Z} K$-module induced from the $\mathbb{Z} K_{1}$-module $H_{1}\left(M_{1}^{\prime}\right)$, and has presentation

$$
(\mathbb{Z} K)^{a_{1}} \stackrel{\Delta_{1}}{\longrightarrow}(\mathbb{Z} K)^{b_{1}} \rightarrow H_{1}\left(X_{1}\right) \otimes_{\mathbb{Z} K_{1}} \mathbb{Z} K \rightarrow 0 .
$$

The second one is the $\mathbb{Z} K$-module induced from the trivial $\mathbb{Z} K_{2}$-module $\mathbb{Z}$, and has presentation

$$
(\mathbb{Z} K)^{n_{2}} \stackrel{D_{2}}{\longrightarrow} \mathbb{Z} K \rightarrow \mathbb{Z} \otimes_{\mathbb{Z} K_{2}} \mathbb{Z} K \rightarrow 0 .
$$

Taking the tensor product (over $\mathbb{Z} K$ ) of the complexes (1.1) and (1.2) and truncating yields the following presentation for the first summand of $H_{1}\left(M^{\prime}\right)$ :

$$
(\mathbb{Z} K)^{a_{1}+n_{2} b_{1}} \stackrel{\left(\begin{array}{c}
\Delta_{1} \\
D_{2}^{b_{1}}
\end{array}\right)}{\longrightarrow}(\mathbb{Z} K)^{b_{1}} \rightarrow\left(H_{1}\left(X_{1}\right) \otimes_{\mathbb{Z} K_{1}} \mathbb{Z} K\right) \otimes_{\mathbb{Z} K_{2}} \mathbb{Z} \rightarrow 0 .
$$

The second summand is handled the same way, and that finishes the proof.

Corollary 1.10. The ranks of the Chen groups of $G=\pi_{1}\left(M_{1} \times M_{2}\right)$ are given by

$$
\theta_{k}(G)=\theta_{k}\left(G_{1}\right)+\theta_{k}\left(G_{2}\right),
$$

where $G_{i}=\pi_{1}\left(M_{i}\right)$.

Example 1.11. Let $G=F_{d_{1}} \times \cdots \times F_{d_{\ell}}$ be a direct product of finitely generated free groups. Using the above result, and the calculation in Example 1.6, one can easily recover the ranks of the Chen groups of $G$ announced in [5]: $\theta_{1}=\sum_{i=1}^{\ell} d_{i}$ and $\theta_{k}=(k-1) \cdot \sum_{i=1}^{\ell}\left(\begin{array}{c}k+d_{i}-2 \\ k\end{array}\right)$ for $k \geq 2$.

Example 1.12. Let $\mathcal{A}$ be an affine arrangement of $n$ hyperplanes in $\mathbb{C}^{d}$, and let $\overline{\mathcal{A}}$ be the cone of $\mathcal{A}$, a central arrangement of $n+1$ hyperplanes in $\mathbb{C}^{d+1}$ (see [27]). It is well-known that the complement $\bar{M}$ of $\overline{\mathcal{A}}$ is homeomorphic to the product of the complement $M$ of $\mathcal{A}$ and $\mathbb{C}^{*}, \bar{M}=M \times \mathbb{C}^{*}$.

Fix a generator $x$ for $\pi_{1}\left(\mathbb{C}^{*}\right)=\mathbb{Z}$. Let $\Lambda=\mathbb{Z}\left[t_{1}^{ \pm 1}, \ldots, t_{n}^{ \pm 1}\right]$, and suppose that the Alexander invariant $B$ of $\mathcal{A}$ has presentation $\Lambda^{a} \stackrel{\Delta}{\longrightarrow} \Lambda^{b} \rightarrow B \rightarrow 0$ (see section 5). Using Theorem 1.9, we obtain a presentation $\bar{\Lambda}^{a+b} \stackrel{\bar{\Delta}}{\longrightarrow} \bar{\Lambda}^{b} \rightarrow \bar{B} \rightarrow 0$ for the Alexander invariant of $\overline{\mathcal{A}}$, where $\bar{\Lambda}=\Lambda\left[x^{ \pm 1}\right]$ and $\bar{\Delta}=(\Delta \quad(x-1) \cdot \mathrm{id})^{\top}$. Thus the ranks of the Chen groups of $G(\overline{\mathcal{A}})$ coincide with those of $G(\mathcal{A})$ for $k \geq 2$. Note that $\theta_{1}(\overline{\mathcal{A}})=\theta_{1}(\mathcal{A})+1=n+1$. 


\section{A QuiCK TRIP THROUgh Fox CALCUlus}

In this section we review the basics of Fox's free differential calculus, as introduced in [17], and developed in [3], and derive some consequences.

2.1. Fox Gradient. Let $F_{n}$ be the free group on generators $t_{1}, \ldots, t_{n}$, and $\mathbb{Z} F_{n}$ its group ring. Let $W_{n}=\bigvee_{1}^{n} S^{1}$ be a wedge of $n$ circles, with basepoint $*$ at the wedge point. Let $\widetilde{W}_{n}$ be the universal cover, and let $\widetilde{C}_{\bullet}\left(\widetilde{W}_{n}\right)$ be the augmented, equivariant chain complex of $\widetilde{W}_{n}$. Identifying $C_{0}\left(\widetilde{W}_{n}\right)$ with $\mathbb{Z} F_{n}$, and $C_{1}\left(\widetilde{W}_{n}\right)$ with the free $\mathbb{Z} F_{n}$-module of rank $n$ (with basis $e_{1}, \ldots, e_{n}$ given by the lifts of the 1-cells at the basepoint $\widetilde{*}$ ), we obtain the standard free $\mathbb{Z} F_{n}$-resolution of $\mathbb{Z}$,

$$
0 \rightarrow\left(\mathbb{Z} F_{n}\right)^{n} \stackrel{\partial_{1}}{\longrightarrow} \mathbb{Z} F_{n} \stackrel{\epsilon}{\rightarrow} \mathbb{Z} \rightarrow 0
$$

where $\partial_{1}\left(e_{i}\right)=t_{i}-1$ and $\epsilon\left(t_{i}\right)=1$. The Fox calculus is based on the observation that the augmentation ideal, $I F_{n}=\operatorname{ker} \epsilon$, is a free $\mathbb{Z} F_{n}$-module of rank $n$, generated by the entries of the matrix of $\partial_{1}$. This can be rephrased as follows: Given any $w \in \mathbb{Z} F_{n}$, there exist unique elements $\frac{\partial w}{\partial t_{i}} \in \mathbb{Z} F_{n}$ (called the Fox derivatives of $w$ ) such that the following "fundamental formula of Fox calculus" holds:

$$
w-\epsilon(w)=\sum_{i=1}^{n} \frac{\partial w}{\partial t_{i}}\left(t_{i}-1\right) .
$$

Define the Fox gradient to be the $\mathbb{Z} F_{n}$-linear homomorphism $\nabla: \mathbb{Z} F_{n} \rightarrow\left(\mathbb{Z} F_{n}\right)^{n}$ given by

$$
\nabla(w)=\sum_{i=1}^{n} \frac{\partial w}{\partial t_{i}} e_{i}
$$

Then, formula $(2.1)$ takes the form $\partial_{1}(\nabla(w))=w-\epsilon(w)$. From this can be deduced the following "product rule" for the Fox gradient: $\nabla(u v)=\nabla(u) \cdot \epsilon(v)+u \cdot \nabla(v)$. In particular, $\nabla\left(z^{-1}\right)=-z^{-1} \nabla(z)$, for $z \in F_{n}$.

Now consider an endomorphism $\alpha: F_{n} \rightarrow F_{n}$. This defines a map $\alpha: W_{n} \rightarrow W_{n}$ (unique up to homotopy). The induced chain map $\alpha_{\bullet}: C_{\bullet}\left(\widetilde{W}_{n}\right) \rightarrow C_{\bullet}\left(\widetilde{W}_{n}\right)$ can be written as

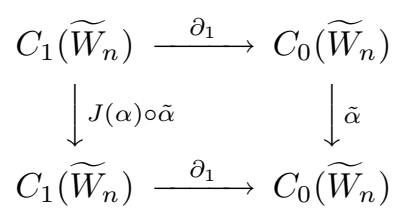

where $J(\alpha):\left(\mathbb{Z} F_{n}\right)^{n} \rightarrow\left(\mathbb{Z} F_{n}\right)^{n}$ is the Fox Jacobian of $\alpha$; namely, the $\mathbb{Z} F_{n}$-linear homomorphism given by $J(\alpha)\left(e_{i}\right)=\nabla\left(\alpha\left(t_{i}\right)\right)$. If $\beta: F_{n} \rightarrow F_{n}$ is another endomorphism, the fact that $(\beta \circ \alpha) \bullet=\beta_{\bullet} \circ \alpha_{\bullet}$ may be rephrased as the "chain rule of Fox calculus:" $J(\alpha \cdot \beta)=\tilde{\beta}(J(\alpha)) \cdot J(\beta)$. In particular, $J(\alpha)^{-1}=\tilde{\alpha} \circ J\left(\alpha^{-1}\right) \circ \tilde{\alpha}^{-1}$.

2.2. Abelianized Fox Jacobian. Let $\mathbb{Z}^{n}$ be the free abelian group on generators $t_{1}, \ldots,[0] t_{n}$, and identify the group $\operatorname{ring} \mathbb{Z}^{n}$ with $\Lambda=\mathbb{Z}\left[t_{1}^{ \pm 1}, \ldots, t_{n}^{ \pm 1}\right]$. Let $T^{n}=$ $\times{ }_{1}^{n} S^{1}$ be the $n$-torus. The augmented, equivariant chain complex, $\widetilde{C} \bullet=\widetilde{C}_{\bullet}\left(\widetilde{T}^{n}\right)$, of the universal (abelian) cover can be written as

$$
0 \rightarrow C_{n} \stackrel{d_{n}}{\longrightarrow} \cdots \rightarrow C_{3} \stackrel{d_{3}}{\longrightarrow} C_{2} \stackrel{d_{2}}{\longrightarrow} C_{1} \stackrel{d_{1}}{\longrightarrow} C_{0} \stackrel{\epsilon}{\longrightarrow} \mathbb{Z} \rightarrow 0 .
$$




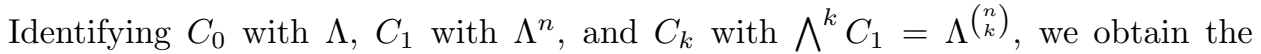
standard free $\Lambda$-resolution of $\mathbb{Z}$. The differentials of this resolution are given by $d_{k}\left(e_{J}\right)=\sum_{r=1}^{k}(-1)^{k+r}\left(t_{j_{r}}-1\right) \cdot e_{J \backslash\left\{j_{r}\right\}}$, where $e_{J}=e_{j_{1}} \wedge \cdots \wedge e_{j_{k}}$ if $J=\left\{j_{1}, \ldots, j_{k}\right\}$.

Let $\mathrm{ab}: F_{n} \rightarrow \mathbb{Z}^{n}, x \mapsto x^{\mathrm{ab}}$, be the abelianization homomorphism. For an element $w \in \mathbb{Z} F_{n}$, let $\nabla^{\mathrm{ab}}(w):=\widetilde{\mathrm{ab}}(\nabla(w)) \in \Lambda^{n}$ be its abelianized Fox gradient. This defines a $\Lambda$-linear homomorphism

$$
\nabla^{\mathrm{ab}}(w): C_{0} \rightarrow C_{1}, \quad u \mapsto \nabla^{\mathrm{ab}}(w) \cdot u .
$$

For an endomorphism $\alpha$ of $F_{n}$, let $\Theta(\alpha):=\widetilde{\mathrm{ab}}(J(\alpha)): C_{1} \rightarrow C_{1}$ be its abelianized Fox Jacobian. This is a $\Lambda$-linear map, whose matrix has rows $\Theta(\alpha)\left(e_{i}\right)=$ $\nabla^{\mathrm{ab}}\left(\alpha\left(t_{i}\right)\right)$. Abelianizing diagram (2.2) yields the chain map:

$$
\begin{aligned}
& C_{1} \stackrel{d_{1}}{\longrightarrow} C_{0} \\
& \downarrow \Theta(\alpha) \circ \tilde{\alpha} \quad \downarrow \tilde{\alpha} \\
& C_{1} \stackrel{d_{1}}{\longrightarrow} C_{0}
\end{aligned}
$$

Set $\Theta_{k}(\alpha)=\bigwedge^{k} \Theta(\alpha): C_{k} \rightarrow C_{k}$ (in particular, $\Theta_{0}=$ id). A computation in the exterior algebra $C_{\bullet}=\wedge^{\bullet} C_{1}$ shows that $\Theta_{k-1}(\alpha) \circ \tilde{\alpha} \circ d_{k}=d_{k} \circ \Theta_{k}(\alpha) \circ \tilde{\alpha}$ for each $k, 1 \leq k \leq n$. Thus, (2.4) extends to a chain map

$$
\Theta \bullet(\alpha) \circ \tilde{\alpha}: C_{\bullet} \rightarrow C_{\bullet}
$$

This chain map is the composite of two chain maps. The first is the (non- $\Lambda$ linear) map $\tilde{\alpha}:\left(C_{\bullet}, d_{\bullet}\right) \rightarrow\left(C_{\bullet}, \tilde{\alpha} \circ d_{\bullet} \circ \tilde{\alpha}^{-1}\right)$. The second is the $(\Lambda$-linear $)$ map $\Theta_{\bullet}(\alpha):\left(C_{\bullet}, \tilde{\alpha} \circ d_{\bullet} \circ \tilde{\alpha}^{-1}\right) \rightarrow\left(C_{\bullet}, d_{\bullet}\right)$.

2.3. Magnus Representations. An automorphism $\alpha \in \operatorname{Aut}\left(F_{n}\right)$ is called an $I A$ automorphism if its abelianization, $\operatorname{ab}(\alpha): \mathbb{Z}^{n} \rightarrow \mathbb{Z}^{n}$, is the identity map. In this case, $\tilde{\alpha}=\mathrm{id}$, and so $\Theta_{\bullet}(\alpha): C_{\bullet} \rightarrow C_{\bullet}$ is a chain map.

The set of IA-automorphisms forms a subgroup of $\operatorname{Aut}\left(F_{n}\right)$, denoted by $\operatorname{IA}\left(F_{n}\right)$. By the chain rule, $\Theta(\alpha \cdot \beta)=\Theta(\alpha) \cdot \Theta(\beta)$, for $\alpha, \beta \in \operatorname{IA}\left(F_{n}\right)$. Thus $\Theta: \operatorname{IA}\left(F_{n}\right) \rightarrow$ $\operatorname{Aut}_{\Lambda}\left(C_{1}\right) \cong \mathrm{GL}(n, \Lambda)$ is a linear representation of $\mathrm{IA}\left(F_{n}\right)$, called the Magnus representation, see [3]. From the above discussion, we see that this representation generalizes to $\Theta_{k}: \operatorname{IA}\left(F_{n}\right) \rightarrow \operatorname{Aut}_{\Lambda}\left(C_{k}\right) \cong \mathrm{GL}\left(\left(\begin{array}{l}n \\ k\end{array}\right), \Lambda\right)$.

Remark 2.4. For $\alpha \in \operatorname{IA}\left(F_{n}\right)$, the chain automorphism $\Theta_{\bullet}(\alpha): C_{\bullet} \rightarrow C_{\bullet}$ admits the following topological interpretation. The map $\alpha: W_{n} \rightarrow W_{n}$ lifts to a map of the maximal abelian covers, $\alpha^{\prime}: W_{n}^{\prime} \rightarrow W_{n}^{\prime}$. View $W_{n}^{\prime}$ as the 1 -skeleton of $\widetilde{T}^{n}$. The map $\alpha^{\prime}$ extends to a $\mathbb{Z}^{n}$-equivariant map $\bar{\alpha}: \widetilde{T}^{n} \rightarrow \widetilde{T}^{n}$. The induced chain map, $\bar{\alpha}_{\bullet}: C_{\bullet}\left(\widetilde{T}^{n}\right) \rightarrow C_{\bullet}\left(\widetilde{T}^{n}\right)$, is chain-equivalent to $\Theta_{\bullet}(\alpha)$.

\section{The Alexander invariant of a free automorphism}

In this section, we find presentations for the Alexander module and the Alexander invariant of the group of an IA-automorphism. A more explicit presentation for the latter is given in case the automorphism is basis-conjugating. 
3.1. The Group of a Free Automorphism. Associated to an automorphism $\alpha$ of the free group $F_{n}=\left\langle t_{1}, \ldots, t_{n}\right\rangle$ is the group

$$
G(\alpha)=\left\langle t_{1}, \ldots, t_{n} \mid t_{1}=\alpha\left(t_{1}\right), \ldots, t_{n}=\alpha\left(t_{n}\right)\right\rangle .
$$

Notice that $\alpha$ induces the identity automorphism on $G(\alpha)$. In fact, $G(\alpha)$ is the maximal quotient of $F_{n}$ with this property. Also, note that $G(\alpha)$ is independent of the choice of free generators for $F_{n}$ : If $x_{1}, \ldots, x_{n}$ is another such choice, then $\left\langle t_{1}, \ldots, t_{n} \mid t_{i}=\alpha\left(t_{i}\right)\right\rangle \cong\left\langle x_{1}, \ldots, x_{n} \mid x_{i}=\alpha\left(x_{i}\right)\right\rangle$. Finally, notice that the group of a free automorphism depends only on the conjugacy class of that automorphism: If $\beta \in \operatorname{Aut}\left(F_{n}\right)$, then

$$
\begin{aligned}
G\left(\beta^{-1} \circ \alpha \circ \beta\right) & =\left\langle t_{i} \mid t_{i}=\beta^{-1} \circ \alpha \circ \beta\left(t_{i}\right)\right\rangle \\
& =\left\langle t_{i} \mid \beta\left(t_{i}\right)=\alpha\left(\beta\left(t_{i}\right)\right)\right\rangle \\
& =\left\langle x_{i} \mid x_{i}=\alpha\left(x_{i}\right)\right\rangle \cong G(\alpha) .
\end{aligned}
$$

See [25] for details.

Topologically, the group $G(\alpha)$ can be interpreted as follows. Recall that $W_{n}$ denotes a wedge of $n$ circles, and that $\alpha: W_{n} \rightarrow W_{n}$ also denotes a basepoint preserving homotopy equivalence that induces $\alpha: F_{n} \rightarrow F_{n}$ on fundamental groups. Let $Y(\alpha)=W_{n} \times_{\alpha} S^{1}$ be the mapping torus of $\alpha$; its fundamental group is the semidirect product $F_{n} \rtimes_{\alpha} \mathbb{Z}=\left\langle t_{1}, \ldots, t_{n}, x \mid x^{-1} t_{i} x=\alpha\left(t_{i}\right)\right\rangle$. Let $X(\alpha)=$ $W_{n} \times{ }_{\alpha} S^{1} \bigcup_{* \times S^{1}} * \times D^{2}$. Then $\pi_{1}(X(\alpha))=G(\alpha)$, and, in fact, $X(\alpha)$ is homotopy equivalent to the 2-complex associated to the above presentation of $G(\alpha)$.

3.2. Alexander Invariants. Let $\alpha$ be an IA-automorphism of $F_{n}$, and $G=G(\alpha)$ the associated group. Then $H_{1}(G)=\mathbb{Z}^{n}$, the free abelian group generated by $t_{1}, \ldots, t_{n}$. Let $p: X^{\prime} \rightarrow X$ be the corresponding (maximal abelian) cover of $X=$ $X(\alpha)$. We call the $\Lambda$-modules $A(\alpha)=H_{1}\left(X^{\prime}, p^{-1}(*)\right)$, resp. $B(\alpha)=H_{1}\left(X^{\prime}\right)$ the Alexander module, resp. Alexander invariant of $G(\alpha)$. We wish to find presentations for these modules.

First consider $Y=W_{n} \times{ }_{\alpha} S^{1}$. The chain complex of its maximal abelian cover is obtained using the Fox calculus as in [6]:

$$
C_{\bullet}\left(Y^{\prime}\right): \quad C_{2}\left(Y^{\prime}\right) \stackrel{\left(\text { id }-x \cdot \Theta(\alpha) d_{1}\right)}{\longrightarrow} C_{1}\left(Y^{\prime}\right) \stackrel{\left(\begin{array}{c}
d_{1} \\
x-1
\end{array}\right)}{\longrightarrow} C_{0}\left(Y^{\prime}\right) \stackrel{\epsilon}{\longrightarrow} \mathbb{Z} \rightarrow 0,
$$

where the chain groups are the modules over $\bar{\Lambda}=\Lambda\left[x^{ \pm 1}\right]$ given by $C_{2}\left(Y^{\prime}\right)=C_{1} \otimes_{\Lambda} \bar{\Lambda}$, $C_{1}\left(Y^{\prime}\right)=\left(C_{1} \oplus C_{0}\right) \otimes_{\Lambda} \bar{\Lambda}$, and $C_{0}\left(Y^{\prime}\right)=C_{0} \otimes_{\Lambda} \bar{\Lambda}$. It follows that the chain complex of the maximal abelian cover of $X=X(\alpha)$ is

$$
C_{\bullet}\left(X^{\prime}\right): \quad C_{1} \stackrel{\text { id }-\Theta(\alpha)}{\longrightarrow} C_{1} \stackrel{d_{1}}{\longrightarrow} C_{0} \stackrel{\epsilon}{\longrightarrow} \mathbb{Z} \rightarrow 0 .
$$

Hence, $A(\alpha)=\operatorname{coker}(\mathrm{id}-\Theta(\alpha))$ and $B(\alpha)=\operatorname{ker}\left(d_{1}\right) / \operatorname{im}(\mathrm{id}-\Theta(\alpha))$. By homological algebra, there exists a chain map from the chain complex (3.1) to the free $\Lambda$-resolution (2.3), extending the identity map of $\mathbb{Z}$ :

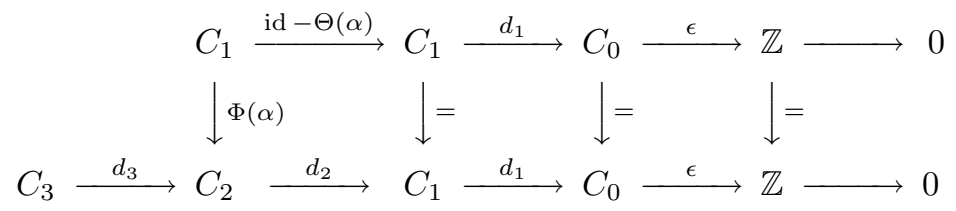

A diagram chase shows that $B(\alpha)=\operatorname{coker}\left(\begin{array}{c}\Phi(\alpha) \\ d_{3}\end{array}\right)$. To summarize, we have: 
Proposition 3.3. If $\alpha \in \operatorname{IA}\left(F_{n}\right)$, the Alexander module and the Alexander invariant of $G(\alpha)$ have presentations

$$
\begin{array}{r}
C_{1} \stackrel{\text { id }-\Theta(\alpha)}{\longrightarrow} C_{1} \rightarrow A(\alpha) \rightarrow 0, \\
C_{1} \oplus C_{3} \stackrel{\left(\Phi(\alpha) d_{3}\right)^{\top}}{\longrightarrow} C_{2} \rightarrow B(\alpha) \rightarrow 0 .
\end{array}
$$

Remark 3.4. The map $\Phi(\alpha)$ is not unique, but rather, it is unique up to chain homotopy: Given two choices, $\Phi_{1}(\alpha)$ and $\Phi_{2}(\alpha)$, there is a homomorphism $D$ : $C_{1} \rightarrow C_{3}$ such that $\Phi_{1}(\alpha)-\Phi_{2}(\alpha)=d_{3} \circ D$. We abbreviate this by saying that $\Phi_{1}(\alpha)=\Phi_{2}(\alpha) \bmod d_{3}$. Of course, any two choices yield equivalent presentations for $B(\alpha)$.

As noted previously, the group $G\left(\beta \circ \alpha \circ \beta^{-1}\right)$ is isomorphic to $G(\alpha)$. The relationship between the corresponding chain maps is as follows:

Proposition 3.5. For $\alpha, \beta \in \operatorname{IA}\left(F_{n}\right)$, we have

$$
\Phi\left(\beta \circ \alpha \circ \beta^{-1}\right)=\Theta_{2}(\beta) \circ \Phi(\alpha) \circ \Theta\left(\beta^{-1}\right) \bmod d_{3} .
$$

Proof. By Remark 3.4, it is enough to show that

$$
d_{2} \circ \Theta_{2}(\beta) \circ \Phi(\alpha) \circ \Theta\left(\beta^{-1}\right)=\mathrm{id}-\Theta\left(\beta \circ \alpha \circ \beta^{-1}\right) .
$$

Since the right-hand side equals $\Theta(\beta) \circ($ id $-\Theta(\alpha)) \circ \Theta\left(\beta^{-1}\right)$, the claim follows from the equalities $d_{2} \circ \Theta_{2}(\beta)=\Theta(\beta) \circ d_{2}$ and $d_{2} \circ \Phi(\alpha)=\mathrm{id}-\Theta(\alpha)$.

3.6. Basis-Conjugating Automorphisms. Let $F_{n}=\left\langle t_{1}, \ldots, t_{n}\right\rangle$. An automorphism $\alpha$ of $F_{n}$ is called a basis-conjugating automorphism if there exists an $n$-tuple $\mathbf{z}=\left(z_{1}, \ldots, z_{n}\right)$, with $z_{i} \in F_{n}$, such that $\alpha=\gamma_{\mathbf{z}}$, where $\gamma_{\mathbf{z}}\left(t_{i}\right)=z_{i} t_{i} z_{i}^{-1}$. The basis-conjugating automorphisms of $F_{n}$ form a subgroup, $\mathrm{CA}\left(F_{n}\right)$, of $\operatorname{Aut}\left(F_{n}\right)$. For $\alpha \in \mathrm{CA}\left(F_{n}\right)$, the following definition/proposition gives an explicit formula for $\Phi(\alpha)$.

Proposition 3.7. For $\gamma_{\mathbf{z}} \in \mathrm{CA}\left(F_{n}\right)$, define the $\Lambda$-homomorphism $\Phi\left(\gamma_{\mathbf{z}}\right): C_{1} \rightarrow$ $C_{2}$ by

$$
\Phi\left(\gamma_{\mathbf{z}}\right)\left(e_{i}\right)=\nabla^{\mathrm{ab}}\left(z_{i}\right) \wedge e_{i}
$$

Then id $-\Theta\left(\gamma_{\mathbf{z}}\right)=d_{2} \circ \Phi\left(\gamma_{\mathbf{z}}\right)$.

Proof. First, note that the Magnus representation of $\gamma_{\mathbf{z}}$ is given by:

$$
\Theta\left(\gamma_{\mathbf{z}}\right)\left(e_{i}\right)=\left(1-t_{i}\right) \cdot \nabla^{\mathrm{ab}}\left(z_{i}\right)+z_{i}^{\mathrm{ab}} \cdot e_{i} .
$$

Hence:

$$
\begin{aligned}
\left(\mathrm{id}-\Theta\left(\gamma_{\mathbf{z}}\right)\right)\left(e_{i}\right) & =\left(t_{i}-1\right) \cdot \nabla^{\mathrm{ab}}\left(z_{i}\right)+\left(1-z_{i}^{\mathrm{ab}}\right) \cdot e_{i} \\
& =d_{1}\left(e_{i}\right) \cdot \nabla^{\mathrm{ab}}\left(z_{i}\right)-d_{1}\left(\nabla^{\mathrm{ab}}\left(z_{i}\right)\right) \cdot e_{i} \\
& =d_{2}\left(\Phi\left(\gamma_{\mathbf{z}}\right)\left(e_{i}\right)\right) .
\end{aligned}
$$

Remark 3.8. As mentioned before, an explicit formula for the Alexander invariant $B(L)$ of an arbitrary link $L \subset S^{3}$ is lacking. If $L$ is a pure link of $n$ components, though, Propositions 3.3 and 3.7 provide a presentation for $B(L)$, with $\left(\begin{array}{l}n \\ 2\end{array}\right)$ generators and $\left(\begin{array}{l}n \\ 3\end{array}\right)+n$ relations. Indeed, as shown by Artin, the braid group $B_{n}$ admits a faithful representation $B_{n} \hookrightarrow \operatorname{Aut}\left(F_{n}\right)$, which restricts to $P_{n} \hookrightarrow \mathrm{CA}\left(F_{n}\right)$. Moreover, any link $L$ is the closure, $\hat{\alpha}$, of a braid $\alpha \in B_{n}$, and $\pi_{1}\left(S^{3} \backslash L\right)=G(\alpha)$. Now assume $L$ is a pure link, i.e, $L=\hat{\alpha}$, for some $\alpha \in P_{n}$. Then $\alpha=\gamma_{\mathbf{z}}$, where $z_{i}$ is the 
longitude corresponding to the meridian $t_{i}$, and we get $B(L)=\operatorname{coker}\left(\begin{array}{c}\Phi\left(\gamma_{\mathbf{z}}\right) \\ d_{3}\end{array}\right)$, with $\Phi\left(\gamma_{\mathbf{z}}\right)$ given by $(3.2)$.

3.9. Alexander Invariant of Several Automorphisms. The above notions generalize in a straightforward manner, from a single automorphism $\alpha$ to several automorphisms $\alpha_{1}, \ldots, \alpha_{s}$ of $F_{n}$. Namely, let

$$
G\left(\alpha_{1}, \ldots, \alpha_{s}\right)=\left\langle t_{1}, \ldots, t_{n} \mid t_{i}=\alpha_{k}\left(t_{i}\right), 1 \leq k \leq s\right\rangle
$$

be the maximal quotient of $F_{n}$ on which all $\alpha_{k}$ act trivially. This group can also be characterized as the quotient of the semidirect product $F_{n} \rtimes F_{s}=\left\langle t_{1}, \ldots, t_{n}, x_{1}, \ldots\right.$, $x_{s}\left|x_{k}^{-1} t_{i} x_{k}=\alpha_{k}\left(t_{i}\right)\right\rangle$ by the normal closure of $F_{s}=\left\langle x_{1}, \ldots, x_{s}\right\rangle$.

Assume $\alpha_{k} \in \operatorname{IA}\left(F_{n}\right)$, for $1 \leq k \leq s$. Let $Y$ be the presentation 2-complex for $F_{n} \rtimes F_{s}$, and $X$ that of $G=G\left(\alpha_{1}, \ldots, \alpha_{s}\right)$. The chain complex of the maximal abelian cover of $Y$ has the following form:

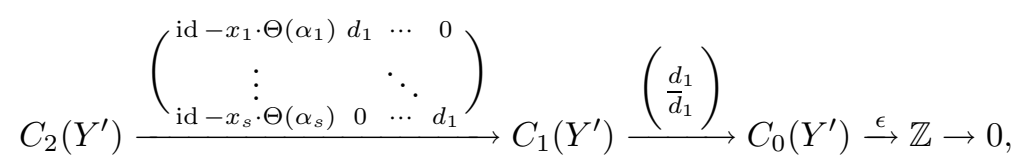

where the chain groups are the modules over $\bar{\Lambda}=\Lambda\left[x_{1}^{ \pm 1}, \ldots, x_{s}^{ \pm 1}\right]$ given by $C_{2}\left(Y^{\prime}\right)=$ $\oplus_{1}^{s} C_{1} \otimes_{\Lambda} \bar{\Lambda}, C_{1}\left(Y^{\prime}\right)=\left(C_{1} \oplus \oplus_{1}^{s} C_{0}\right) \otimes_{\Lambda} \bar{\Lambda}$, and $C_{0}\left(Y^{\prime}\right)=C_{0} \otimes_{\Lambda} \bar{\Lambda}$, and where $\bar{d}_{1}=\left(x_{1}-1 \cdots x_{s}-1\right)^{\top}$. The chain complex of the maximal abelian cover of $X$ is then

$$
C_{1}^{s} \stackrel{\left(\begin{array}{l}
\text { id }-\Theta\left(\alpha_{1}\right) \\
\text { id }-\dddot{\Theta}\left(\alpha_{s}\right)
\end{array}\right)}{\longrightarrow} C_{1} \stackrel{d_{1}}{\longrightarrow} C_{0} \stackrel{\epsilon}{\longrightarrow} \mathbb{Z} \rightarrow 0 .
$$

This chain complex provides a presentation matrix - the so-called Alexander matrix, (id $-\Theta\left(\alpha_{1}\right) \cdots$ id $\left.-\Theta\left(\alpha_{s}\right)\right)^{\top}$-for the Alexander module $A=A\left(\alpha_{1}, \ldots, \alpha_{s}\right)$. Furthermore, if $\Phi\left(\alpha_{k}\right): C_{1} \rightarrow C_{2}$ satisfy $d_{2} \circ \Phi\left(\alpha_{k}\right)=$ id $-\Theta\left(\alpha_{k}\right)$, then the Alexander invariant $B=B\left(\alpha_{1}, \ldots, \alpha_{s}\right)$ has presentation matrix $\left(\Phi\left(\alpha_{1}\right) \cdots \Phi\left(\alpha_{s}\right) d_{3}\right)^{\top}$. When $\alpha_{k} \in \mathrm{CA}\left(F_{n}\right)$, we obtain an explicit presentation for $B$.

Theorem 3.10. Let $\gamma_{\mathbf{z}^{1}}, \ldots, \gamma_{\mathbf{z}^{s}}$ be a collection of basis-conjugating automorphisms of $F_{n}$. Let $\Phi\left(\gamma_{\mathbf{z}^{k}}\right): C_{1} \rightarrow C_{2}$ be the homomorphisms defined by (3.2). Then the Alexander invariant of $G\left(\gamma_{\mathbf{z}^{1}}, \ldots, \gamma_{\mathbf{z}^{s}}\right)$ has presentation

$$
C_{1}^{s} \oplus C_{3} \stackrel{\left(\Phi\left(\gamma_{\mathbf{z}^{1}}\right) \cdots \Phi\left(\gamma_{\mathbf{z}^{s}}\right) d_{3}\right)^{\top}}{\longrightarrow} C_{2} \rightarrow B \rightarrow 0
$$

\section{Local Alexander invariants}

We now find presentations for the Alexander invariant of the group of a full-twist braid automorphism, and that of a related "vertex" group. These presentations are given in terms of the Gassner representation, $\Theta: P_{n} \rightarrow \operatorname{GL}(n, \Lambda)$, which is simply the restriction of the Magnus representation, $\Theta: \operatorname{IA}\left(F_{n}\right) \rightarrow \mathrm{GL}(n, \Lambda)$, to the pure braid group $P_{n}$.

4.1. Alexander Invariant of a Twist Automorphism. Let $V=\left\{i_{1}, \ldots, i_{r}\right\}$ be an increasingly ordered subset of $[n]=\{1, \ldots, n\}$. Let $A_{V}$ be the pure braid in $P_{n}$ which performs a full twist on the strands corresponding to $V$, leaving the other strands fixed. Let $\sigma_{i}(1 \leq i<n)$ be the standard generators of $B_{n}$, and 
$A_{i, j}=\sigma_{j-1} \cdots \sigma_{i+1} \sigma_{i}^{2} \sigma_{i+1}^{-1} \cdots \sigma_{j-1}^{-1}(1 \leq i<j \leq n)$ the standard generators of $P_{n}$, see [3]. The twist on $V$ is given by

$$
A_{V}=\left(A_{i_{1}, i_{2}}\right)\left(A_{i_{1}, i_{3}} A_{i_{2}, i_{3}}\right)\left(A_{i_{1}, i_{4}} A_{i_{2}, i_{4}} A_{i_{3}, i_{4}}\right) \cdots\left(A_{i_{1}, i_{r}} \cdots A_{i_{r-1}, i_{r}}\right) .
$$

A computation with the Artin representation reveals that $A_{V}=\gamma_{\mathbf{w}}$, where $\mathbf{w}=$ $\left(w_{1}, \ldots, w_{n}\right)$ is defined as follows:

$$
w_{i}= \begin{cases}t_{V} & \text { if } i \in V, \\ {\left[t_{V^{i}}, t_{i}\right]} & \text { if } i \in \bar{V} \backslash V, \\ 1 & \text { otherwise, }\end{cases}
$$

where $\bar{V}=\left\{i \in[n] \mid i_{1} \leq i \leq i_{r}\right\}, V^{i}=\{j \in V \mid j<i\},{ }^{i} V=\{j \in V \mid i<j\}$, and $t_{V}=\prod_{j \in V} t_{j}=t_{i_{1}} \cdots t_{i_{r}}$.

Let $G\left(A_{V}\right)=\left\langle t_{1}, \ldots, t_{n} \mid A_{V}\left(t_{i}\right)=t_{i}\right\rangle$ be the group associated to $A_{V} \in \operatorname{Aut}\left(F_{n}\right)$. A computation with (4.2) shows that

$$
G\left(A_{V}\right)=\left\langle t_{1}, \ldots, t_{n} \mid t_{V} t_{i} t_{V}^{-1}=t_{i}, i \in V\right\rangle,
$$

and so $G\left(A_{V}\right) \cong\left(F_{r-1} \times F_{1}\right) * F_{n-r}$. By Proposition 3.3, the Alexander invariant of $G\left(A_{V}\right)$ has presentation

$$
C_{1} \oplus C_{3} \stackrel{\left(\Phi\left(A_{V}\right) d_{3}\right)^{\top}}{\longrightarrow} C_{2} \rightarrow B\left(A_{V}\right) \rightarrow 0
$$

By Proposition 3.7, and a Fox calculus computation, the map $\Phi_{V}:=\Phi\left(A_{V}\right): C_{1} \rightarrow$ $\mathrm{C}_{2}$ is given by

$$
\Phi_{V}\left(e_{i}\right)= \begin{cases}\nabla_{V} \wedge e_{i} & \text { if } i \in V \\ \left(1-t_{i}\right) \nabla_{V} \wedge \nabla_{i_{V}} & \text { if } i \in \bar{V} \backslash V \\ 0 & \text { otherwise. }\end{cases}
$$

where $\nabla_{V}:=\nabla^{\mathrm{ab}}\left(t_{V}\right)=\sum_{i \in V} t_{V^{i}} e_{i}$.

4.2. Simplified Presentation for $B\left(A_{V}\right)$. Set $C_{k}(V)=\operatorname{span}\left\{e_{J} \mid J \subset V\right\}$, and let $\iota_{V}: C_{k}(V) \rightarrow C_{k}$ be the inclusion, and $\pi_{V}: C_{k} \rightarrow C_{k}(V)$ the natural projection. Write $V^{\prime}:=V \backslash\{\min V\}=\left\{i_{2}, \ldots, i_{r}\right\}$. From (4.4) it is apparent that $\Phi_{V}\left(C_{1}\right)=\Phi_{V}\left(C_{1}(V)\right) \subset C_{2}(V)$. Since $0=\nabla_{V} \wedge \nabla_{V}=\nabla_{V} \wedge e_{i_{1}}+\nabla_{V} \wedge \sum_{i \in V^{\prime}} t_{V^{i}} e_{i}$, we see that $\Phi_{V}\left(e_{i_{1}}\right) \in \Phi_{V}\left(C_{1}\left(V^{\prime}\right)\right)$. Thus,

$$
\Phi_{V}\left(C_{1}\right)=\Phi_{V}\left(C_{1}\left(V^{\prime}\right)\right) \subset C_{2}(V) .
$$

Define an automorphism $\mu_{V}: F_{n} \rightarrow F_{n}$ by:

$$
\mu_{V}\left(t_{i}\right)= \begin{cases}t_{V} & \text { if } i=\min V \\ t_{i} & \text { otherwise. }\end{cases}
$$

Note that $\Theta\left(\mu_{V}\right)\left(e_{i_{1}}\right)=\nabla_{V}$, and $\Theta_{2}\left(\mu_{V}\right)\left(e_{i_{1}} \wedge e_{i}\right)=\Phi_{V}\left(e_{i}\right)$, for $i \in V^{\prime}$. Thus,

$$
\Theta_{2}\left(\mu_{V}\right)^{-1} \circ \Phi_{V}\left(e_{i}\right)=e_{i_{1}} \wedge e_{i} \text { for } i \in V^{\prime} .
$$

Let $C_{2}^{\prime}(V)$ be the direct summand of $C_{2}$ spanned by $\left\{e_{i_{1}} \wedge e_{i} \mid i \in V^{\prime}\right\}$, let $C_{2}^{\perp}(V)$ be the complementary summand, and let $\pi^{V}: C_{2} \rightarrow C_{2}^{\perp}(V)$ be the canonical projection. Putting together (4.3), (4.5), and (4.6), we obtain: 
Proposition 4.3. The Alexander invariant of $G\left(A_{V}\right)$ has presentation

$$
C_{3} \stackrel{\Delta(V)}{\longrightarrow} C_{2}^{\perp}(V) \rightarrow B\left(A_{V}\right) \rightarrow 0,
$$

where $\Delta(V)=\pi^{V} \circ \Theta_{2}\left(\mu_{V}\right)^{-1} \circ d_{3}$.

4.4. Alexander Invariant of a Vertex Group. To the twist automorphism $A_{V}$, we also associate a "vertex group," $G_{V}:=G\left(\left\{A_{V}, A_{i, j}||\{i, j\} \cap V \mid \leq 1\right\}\right)$. Using (4.2), we obtain the following presentation:

$$
\left.G_{V}=\left\langle t_{1}, \ldots, t_{n}\right| t_{V} t_{i} t_{V}^{-1}=t_{i} \text { if } i \in V, \quad t_{j} t_{i} t_{j}^{-1}=t_{i} \text { if }\{i, j\} \not \subset V\right\rangle .
$$

Remark 4.5. The group $G_{V}$ has the following geometric interpretation. Let $\mathcal{A}_{V}=$ $\left\{H_{1}, \ldots, H_{n}\right\}$ be an arrangement of $n$ distinct lines in $\mathbb{C}^{2}$ such that $H_{i}$ passes through 0 for $i \in V$, and $H_{j}$ is in general position with $H_{k}$, for $j \notin V$, and $k \neq j$. Then $G_{V}$ is isomorphic to the group of the arrangement $\mathcal{A}_{V}: G_{V} \cong$ $\pi_{1}\left(\mathbb{C}^{2} \backslash \bigcup_{i=1}^{n} H_{i}\right)$.

A (minimal) presentation for the Alexander invariant $B_{V}=B\left(G_{V}\right)$ may be obtained from (4.7) by restricting the map $\Delta(V)$ to a map $C_{2}\left(V^{\prime}\right) \wedge C_{1} \rightarrow C_{2}\left(V^{\prime}\right)$, and some further matrix operations. Alternatively, it may be obtained by applying Theorem 1.9 to the direct product decomposition $G_{V} \cong F_{r-1} \times \mathbb{Z}^{n-r+1}$, apparent from (4.8). The result is as follows:

Proposition 4.6. The Alexander invariant of $G_{V}$ has presentation

$$
C_{2}\left(V^{\prime}\right) \wedge C_{1} \stackrel{\Delta_{V}}{\longrightarrow} C_{2}\left(V^{\prime}\right) \rightarrow B_{V} \rightarrow 0,
$$

where $\Delta_{V}=\pi_{V^{\prime}} \circ \tilde{\mu}_{V} \circ d_{3} \circ \tilde{\mu}_{V}^{-1} \circ\left(\iota_{V^{\prime}} \wedge \mathrm{id}\right)$.

(This will be useful only when $|V| \geq 3$; if $|V|=2$, then $G_{V}=\mathbb{Z}^{n}, B_{V}=0$, and $C_{2}\left(V^{\prime}\right)=0$.)

The above presentation may be extended to a free resolution,

$$
\cdots \rightarrow C_{2}\left(V^{\prime}\right) \wedge C_{2} \stackrel{\Delta_{V}^{2}}{\longrightarrow} C_{2}\left(V^{\prime}\right) \wedge C_{1} \stackrel{\Delta_{V}}{\longrightarrow} C_{2}\left(V^{\prime}\right) \rightarrow B_{V} \rightarrow 0,
$$

with boundary maps $\Delta_{V}^{\bullet}$ given by

$$
\Delta_{V}^{k}=\left(\pi_{V^{\prime}} \wedge \mathrm{id}\right) \circ \tilde{\mu}_{V} \circ d_{k+2} \circ \tilde{\mu}_{V}^{-1} \circ\left(\iota_{V^{\prime}} \wedge \mathrm{id}\right): C_{2}\left(V^{\prime}\right) \wedge C_{k} \rightarrow C_{2}\left(V^{\prime}\right) \wedge C_{k-1} .
$$

Furthermore, by the discussion following (2.5), there exists a naturally defined chain map $\Psi_{V, \bullet}:\left(C_{\bullet}, d_{\bullet}\right) \rightarrow\left(C_{2}\left(V^{\prime}\right) \wedge C_{\bullet-2}, \Delta_{V}^{\bullet-2}\right)$, given by

$$
\Psi_{V, k}=\left(\pi_{V^{\prime}} \wedge \mathrm{id}\right) \circ \Theta_{k}\left(\mu_{V}\right)^{-1}: C_{k} \rightarrow C_{2}\left(V^{\prime}\right) \wedge C_{k-2}, \quad \text { for } k \geq 2 .
$$

\section{The Alexander invariant of an arrangement}

In this section, we use the results of the previous sections to obtain a presentation for the Alexander invariant of the group of a hyperplane arrangement.

5.1. Braid Monodromy. The fundamental group of the complement of an arrangement of complex hyperplanes is, by a Lefschetz-type theorem of Zariski ([19], [18]), isomorphic to that of a generic two-dimensional section. So, for the purpose of computing the Alexander invariant, it is enough to consider affine line arrangements in $\mathbb{C}^{2}$. Let $\mathcal{A}=\left\{H_{1}, \ldots, H_{n}\right\}$ be such an arrangement, with vertices 
$\mathcal{V}=\left\{v_{1}, \ldots, v_{s}\right\}$. If $v_{k}=H_{i_{1}} \cap \cdots \cap H_{i_{r}}$, let $V_{k}=\left\{i_{1}, \ldots, i_{r}\right\}$ denote the corresponding "vertex set." We identify the set $L_{2}(\mathcal{A})$ of rank two elements in the lattice of $\mathcal{A}$ and the collection $\left\{V_{1}, \ldots, V_{s}\right\}$ of vertex sets of $\mathcal{A}$.

The braid monodromy of $\mathcal{A}$ is determined as follows (see [7] for details). Choose coordinates $(x, z)$ in $\mathbb{C}^{2}$ so that the projection $\operatorname{pr}_{1}: \mathbb{C}^{2} \rightarrow \mathbb{C}$ is generic with respect to $\mathcal{A}$. Let $f(x, z)=\prod_{i=1}^{n}\left(z-a_{i}(x)\right)$ be a defining polynomial for $\mathcal{A}$. The root map $a=\left(a_{1}, \ldots, a_{n}\right): \mathbb{C} \rightarrow \mathbb{C}^{n}$ restricts to a map from the complement of $\mathcal{Y}=\operatorname{pr}_{1}(\mathcal{V})$ to the complement of the braid arrangement $\mathcal{A}_{n}=\left\{\operatorname{ker}\left(y_{i}-y_{j}\right)\right\}_{1 \leq i<j \leq n}$. Identify $\pi_{1}(\mathbb{C} \backslash \mathcal{Y})$ with the free group $F_{s}=\left\langle x_{1}, \ldots, x_{s}\right\rangle$, and $\pi_{1}\left(\mathbb{C}^{n} \backslash \bigcup_{H \in \mathcal{A}_{n}} H\right)$ with the pure braid group $P_{n}$. Then, the braid monodromy of $\mathcal{A}$ is the induced homomorphism on fundamental groups, $\alpha: F_{s} \rightarrow P_{n}$.

The braid monodromy generators $\alpha_{k}=\alpha\left(x_{k}\right)$ can be written explicitly using a braided wiring diagram $\mathcal{W}$ associated to $\mathcal{A}$. Such a diagram, determined by the choices made above, may be (abstractly) specified by a sequence of vertex sets and braids, $\mathcal{W}=\mathcal{W}_{s}=\left\{V_{1}, \beta_{1}, V_{2}, \beta_{2}, \ldots, \beta_{s-1}, V_{s}\right\}$. The braid monodromy generators are given by $\alpha_{k}=A_{V_{k}}^{\delta_{k}}$, where $A_{V_{k}}$ is the twist braid defined in (4.1) and $\delta_{k}$ is a pure braid determined by the subdiagram $\mathcal{W}_{k}$.

5.2. The Presentation for $B(\mathcal{A})$. Let $M=M(\mathcal{A})$ be the complement of $\mathcal{A}$. Let $G=G\left(\alpha_{1}, \ldots, \alpha_{s}\right)$ be the fundamental group of $M$, with Alexander invariant $B=B(\mathcal{A})$. Theorem 3.10 provides the following presentation for $B$ :

$$
C_{1}^{s} \oplus C_{3} \stackrel{\left(\Phi_{V_{1}}^{\delta_{1}} \cdots \Phi_{V_{s}}^{\delta_{s}} d_{3}\right.}{{ }^{\top}} C_{2} \rightarrow B \rightarrow 0
$$

where $\Phi_{V}^{\delta}:=\Phi\left(A_{V}^{\delta}\right)=\Theta_{2}(\delta) \circ \Phi_{V} \circ \Theta\left(\delta^{-1}\right): C_{1} \rightarrow C_{2}$, and $\Phi_{V}$ is given by (4.4).

This presentation can be simplified, based on the following elementary observation: If $R$ is a ring, and $B$ is an $R$-module, with presentation $R^{p} \stackrel{\Delta}{\longrightarrow} R^{q} \rightarrow B \rightarrow 0$, where $\Delta=\Upsilon \circ \Xi$, or $\Delta=\Xi \circ \Upsilon$, with $\Xi$ invertible, then $B$ can also be presented as $R^{p} \stackrel{\Upsilon}{\rightarrow} R^{q} \rightarrow B \rightarrow 0$. Since the maps $\Theta\left(\delta_{k}^{-1}\right)$ are invertible we may replace $\Phi_{V_{k}}^{\delta_{k}}$ by $\Theta_{2}\left(\delta_{k}\right) \circ \Phi_{V_{k}}$. Furthermore, by (4.5), we may subsequently restrict each of the maps $\Phi_{V_{k}}$ to $\Phi_{k}: C_{1}\left(V_{k}^{\prime}\right) \rightarrow C_{2}$. Thus, we obtain the following:

Theorem 5.3. The Alexander invariant of an arrangement $\mathcal{A}$, with braid monodromy generators $A_{V_{1}}^{\delta_{1}}, \ldots, A_{V_{s}}^{\delta_{s}}$, has presentation

$$
K_{1} \stackrel{\Delta}{\longrightarrow} K_{0} \rightarrow B(\mathcal{A}) \rightarrow 0
$$

where $K_{1}=\bigoplus_{k=1}^{s} C_{1}\left(V_{k}^{\prime}\right) \oplus C_{3}, K_{0}=C_{2}$, and $\Delta=\left(\begin{array}{ll}\Phi & d_{3}\end{array}\right)^{\top}$, with $\left.\Phi\right|_{C_{1}\left(V_{k}^{\prime}\right)}=$ $\Theta_{2}\left(\delta_{k}\right) \circ \Phi_{k}$.

Note that this presentation has $\left(\begin{array}{l}n \\ 2\end{array}\right)$ generators and $\sum_{k=1}^{s}\left(\left|V_{k}\right|-1\right)+\left(\begin{array}{c}n \\ 3\end{array}\right)$ relations, and that $\sum_{k=1}^{s}\left(\left|V_{k}\right|-1\right)=b_{2}(M)$.

5.4. Real Arrangements. The presentation can be simplified in the case where $\mathcal{A}$ is the complexification of a line arrangement $\mathcal{A}_{\mathbb{R}}$ in $\mathbb{R}^{2}$. In this instance, the wiring diagram $\mathcal{W}$ can be chosen so that it contains no intermediary braids, and each "conjugating braid," $\delta_{k}$, is a subword of the full twist, $A_{[n]}$, on $n$ strands. 
Let $U_{k}$ denote the set of indices of wires of $\mathcal{W}$ which lie above the vertex $v_{k}$ in $\operatorname{pr}_{1}^{-1}\left(y_{k}\right)$, and let $J_{k}=\left(\bar{V}_{k} \backslash V_{k}\right) \cap U_{k}$. Then the conjugating braids may be written as $\delta_{k}=\prod_{j<i} A_{j, i}$, where the product is over all $i \in V_{k}$ and $j \in J_{k}$, see [8], [7].

Define a homomorphism $\Theta_{2}(\mu): C_{2} \rightarrow C_{2}$ by

$$
\boldsymbol{\Theta}_{2}(\mu)\left(e_{i} \wedge e_{j}\right)= \begin{cases}\Theta_{2}\left(\mu_{V_{k}}\right)\left(e_{i} \wedge e_{j}\right) & \text { if }\{i, j\} \subset V_{k}, \\ e_{i} \wedge e_{j} & \text { otherwise. }\end{cases}
$$

It is readily seen that $\boldsymbol{\Theta}_{2}(\mu)$ is invertible. Similarly, define $\boldsymbol{\Theta}_{2}(\delta): C_{2} \rightarrow C_{2}$. A computation shows that $\delta_{k}\left(t_{i}\right)=t_{J_{k}^{i}} \cdot t_{i} \cdot t_{J_{k}^{i}}^{-1}$, and that $\Theta\left(\delta_{k}\right)\left(e_{i}\right)=\left(1-t_{i}\right) \cdot \nabla_{J_{k}^{i}}+$ $t_{J_{k}^{i}} \cdot e_{i}$, for $i \in V_{k}$. Thus, $\boldsymbol{\Theta}_{2}(\delta)$ is also invertible. Proceeding as in 4.2, we obtain the following.

Theorem 5.5. The Alexander invariant of a complexified real arrangement $\mathcal{A}$ has presentation

$$
C_{3} \stackrel{\Delta^{\prime}}{\longrightarrow} L_{0} \rightarrow B(\mathcal{A}) \rightarrow 0
$$

where $L_{0}$ is the complementary summand to $K_{0}^{\prime}=\bigoplus_{V} C_{2}^{\prime}(V)$ in $C_{2}, \pi_{0}: C_{2} \rightarrow L_{0}$ is the canonical projection, and $\Delta^{\prime}=\pi_{0} \circ \boldsymbol{\Theta}_{2}(\mu)^{-1} \circ \boldsymbol{\Theta}_{2}(\delta)^{-1} \circ d_{3}$.

Note that this presentation has only $\left(\begin{array}{l}n \\ 2\end{array}\right)-b_{2}(M)$ generators, and $\left(\begin{array}{l}n \\ 3\end{array}\right)$ relations.

Remark 5.6. For an arbitrary complex arrangement, the map $\boldsymbol{\Theta}_{2}(\delta)$ need not be invertible. Thus the simplification of the presentation of the Alexander invariant afforded by the above result may not be available. However, for any arrangement, we obtain an analogous simplified presentation for the $I$-adic completion, $\widehat{B}(\mathcal{A})$, of the Alexander invariant of $\mathcal{A}$ in Corollary 6.6.

\section{Decomposition of the Alexander invariant}

We now relate the Alexander invariant of an arrangement $\mathcal{A}$ to a "combinatorial" Alexander invariant, determined by the intersection lattice of $\mathcal{A}$. For these purposes, we restrict our attention to central arrangements and their generic sections. It is enough to consider an affine arrangement, $\mathcal{A}=\left\{H_{1}, \ldots, H_{n}\right\}$, of $n$ lines in $\mathbb{C}^{2}$ that is transverse to infinity (that is, no two lines of $\mathcal{A}$ are parallel). Recall that we identify the set of rank two elements in the lattice of $\mathcal{A}$ and the collection of vertex sets of $\mathcal{A}: L_{2}(\mathcal{A})=\left\{V_{1}, \ldots, V_{s}\right\}$.

6.1. The Coarse Combinatorial Alexander Invariant. For each $V \in L_{2}(\mathcal{A})$, let $\mathcal{A}_{V}=\left\{H_{V, 1}, \ldots, H_{V, n}\right\}$ denote the arrangement obtained from $\mathcal{A}$ by perturbing the lines so that all lines except those passing through the corresponding vertex $v$ are in general position. Let $\mathcal{A}^{\mathrm{cc}}=\prod_{V} \mathcal{A}_{V}$ be the product of these arrangements (see [27]). Define the coarse combinatorial Alexander invariant of $\mathcal{A}$ to be the module $B^{\text {cc }}(\mathcal{A})=B\left(\mathcal{A}^{\text {cc }}\right) \otimes_{\mathbb{Z}^{n s}} \mathbb{Z}^{n}$ induced from the Alexander invariant of $\mathcal{A}^{\text {cc }}$ by the projection $t_{V, j} \mapsto t_{j}$.

By Remark 4.5, the group of $\mathcal{A}_{V}$ is isomorphic to the vertex group $G_{V}$. Let $B_{V}$ be the Alexander invariant of $G_{V}$, as determined in 4.4. By Theorem 1.9, the coarse combinatorial Alexander invariant of $\mathcal{A}$ decomposes as a direct sum of Alexander invariants of vertex groups:

$$
B^{\mathrm{cc}}(\mathcal{A})=\bigoplus_{V \in L_{2}(\mathcal{A})} B_{V}
$$


Our goal in this subsection is to define an epimorphism $\Pi: B \rightarrow B^{\mathrm{cc}}$ from the Alexander invariant of the arrangement $\mathcal{A}$ to that of its "coarse combinatorial approximation."

Notice that the module $B_{V}$ depends only on the cardinality $|V|$ of the vertex set $V$. Consequently, the module $B^{\mathrm{cc}}$ depends only on the number and multiplicities of the elements of $L_{2}(\mathcal{A})$. This $\Lambda$-module admits a free resolution

$$
\cdots \rightarrow L_{2} \stackrel{D_{2}}{\longrightarrow} L_{1} \stackrel{D_{1}}{\longrightarrow} L_{0} \rightarrow B^{\mathrm{cc}} \rightarrow 0
$$

obtained by taking the direct sum of the resolutions (4.9): $L_{k}=\bigoplus_{V} C_{2}\left(V^{\prime}\right) \wedge$ $C_{k}, D_{k}=\bigoplus_{V} \Delta_{V}^{k}$. (Since $\mathcal{A}$ is assumed to be transverse to infinity, $L_{0}=$ $\bigoplus_{V} C_{2}\left(V^{\prime}\right)$ is indeed the complementary summand of $K_{0}^{\prime}=\bigoplus_{V} C_{2}^{\prime}(V)$ in $K_{0}=$ $C_{2}=\bigoplus_{V} C_{2}(V)$.)

Let $\Psi_{V, \bullet}: C_{\bullet} \rightarrow C_{2}\left(V^{\prime}\right) \wedge C_{\bullet-2}$ be the chain map introduced in (4.10). Define a chain map $\Psi_{\bullet}: C_{\bullet} \rightarrow L_{\bullet}-2$ by $\Psi_{k}=\sum_{V} \Psi_{V, k}$, for $k \geq 2$.

Proposition 6.2. The image of the composition $\Psi_{2} \circ \Phi: \bigoplus_{V} C_{1}\left(V^{\prime}\right) \rightarrow L_{0}$ is contained in the image of the map $D_{1}: L_{1} \rightarrow L_{0}$. Therefore, there exists a map $\Gamma: \bigoplus_{V} C_{1}\left(V^{\prime}\right) \rightarrow L_{1}$ such that $D_{1} \circ \Gamma=\Psi_{2} \circ \Phi$.

Proof. Let $A_{V}^{\delta}$ be a braid monodromy generator of $\mathcal{A}$, where $V=\left\{i_{1}, \ldots, i_{r}\right\}$ and $\delta$ is some pure braid. Using the pure braid relations to rewrite $\delta$ if necessary, we may assume that this pure braid is a word in the generators $\left\{A_{r, s} \mid\{r, s\} \not \subset V\right\}$.

For $j \in V^{\prime}$, we have $\Psi_{2} \circ \Phi\left(e_{j}\right)=\Psi_{2} \circ \Theta_{2}(\delta)\left(\nabla_{V} \wedge e_{j}\right)$. Since $\delta \in \operatorname{IA}\left(F_{n}\right)$, we have $\operatorname{im}\left(\Theta_{k}(\delta)-\mathrm{id}\right) \subset I \cdot C_{k}$. Hence, $\Theta_{2}(\delta)\left(e_{i} \wedge e_{j}\right)=e_{i} \wedge e_{j}+W_{i, j}^{\delta}$, where $W_{i, j}^{\delta}=$ $\sum w_{p, q} e_{p} \wedge e_{q}$, with $w_{p, q} \in I$. Thus $\Psi_{2} \circ \Phi\left(e_{j}\right)=\Psi_{2}\left(\nabla_{V} \wedge e_{j}\right)+\sum_{i \in V} t_{V^{i}} \cdot \Psi_{2}\left(W_{i, j}^{\delta}\right)$. Since $\Psi_{2}\left(\nabla_{V} \wedge e_{j}\right)=\pi_{V^{\prime}} \circ \Theta_{2}\left(\mu_{V}\right)^{-1}\left(\Theta\left(\mu_{V}\right)\left(e_{i_{1}} \wedge e_{j}\right)\right)=\pi_{V^{\prime}}\left(e_{i_{1}} \wedge e_{j}\right)=0$, it suffices to show that

$$
\Psi_{2}\left(W_{i, j}^{\delta}\right) \in \operatorname{im}\left(D_{1}\right)
$$

For a vertex set $U \in L_{2}(\mathcal{A})$, recall the natural projection $\pi_{U}: C_{k} \rightarrow C_{k}(U)$, and denote by $I_{U}^{\perp}$ the ideal in $\Lambda$ generated by $\left\langle 1-t_{k} \mid k \notin U\right\rangle$.

Claim. For each vertex set $U \in L_{2}(\mathcal{A})$, we have $\pi_{U}\left(W_{i, j}^{\delta}\right) \in I_{U}^{\perp} \cdot C_{2}(U)$.

Before proving this claim, let us show that it implies (6.1). For $1 \leq p<q \leq n$, let $V(p, q)$ denote the unique vertex set of $\mathcal{A}$ with $p, q \in V(p, q)$. If $w \cdot e_{p} \wedge e_{q}$ is a summand of $W_{i, j}^{\delta}$, write $U=V(p, q)$. Then, by the claim, we have $w \in I_{U}^{\perp}$. Now $\Psi_{2}\left(e_{p} \wedge e_{q}\right)=\Psi_{U, 2}\left(e_{p} \wedge e_{q}\right) \in C_{2}\left(U^{\prime}\right)$, and it is readily checked that $I_{U}^{\perp} \cdot C_{2}\left(U^{\prime}\right) \subset$ $\operatorname{im}\left(\Delta_{U}\right)$. It follows that $\Psi_{2}\left(w \cdot e_{p} \wedge e_{q}\right) \in \operatorname{im}\left(\Delta_{U}\right)$.

Thus it suffices to prove the claim. This may be accomplished by induction on the length of the word $\delta$. If $\delta=1$, then $W_{i, j}^{\delta}=0$, and there is nothing to prove. If $\delta=A_{r, s}^{ \pm 1}$, a computation shows that $\Theta_{2}\left(A_{r, s}\right)\left(e_{i} \wedge e_{j}\right)=e_{i} \wedge e_{j}+W_{i, j}^{r, s}$ and 


$$
\begin{aligned}
& \Theta_{2}\left(A_{r, s}^{-1}\right)\left(e_{i} \wedge e_{j}\right)=e_{i} \wedge e_{j}-\left(t_{r} t_{s}\right)^{-1} W_{i, j}^{r, s}, \text { where } \\
& W_{i, j}^{r, s}= \begin{cases}t_{r}\left(t_{s}-1\right) e_{i} \wedge e_{r}+t_{r}\left(1-t_{r}\right) e_{i} \wedge e_{s} & \text { if } i<j=r<s, \\
\left(1-t_{j}\right)\left[\left(1-t_{s}\right) e_{i} \wedge e_{r}+\left(t_{r}-1\right) e_{i} \wedge e_{s}\right] & \text { if } i<r<j<s, \\
\left(t_{r}-1\right) e_{i} \wedge e_{s}+\left(1-t_{s}\right) e_{i} \wedge e_{r} & \text { if } i<r<j=s, \\
\left(t_{r} t_{s}-1\right) e_{r} \wedge e_{s} & \text { if } i=r<j=s, \\
\left(t_{r}-1\right) e_{i} \wedge e_{s}+\left(t_{s}-1\right)\left[e_{r} \wedge e_{i}+\left(t_{i}-1\right) e_{r} \wedge e_{s}\right] & \text { if } r<i<j=s, \\
\left(1-t_{i}\right)\left[\left(1-t_{s}\right) e_{r} \wedge e_{j}+\left(t_{r}-1\right) e_{s} \wedge e_{j}\right] & \text { if } r<i<s<j, \\
t_{r}\left(t_{s}-1\right) e_{r} \wedge e_{j}+t_{r}\left(1-t_{r}\right) e_{s} \wedge e_{j} & \text { if } r=i<s<j, \\
\left(t_{r}-1\right) e_{s} \wedge e_{j}+\left(1-t_{s}\right) e_{r} \wedge e_{j} & \text { if } r<i=s<j, \\
t_{r}\left(t_{s}-1\right) e_{r} \wedge e_{j}+\left(t_{r}-1\right)\left[t_{r} e_{j} \wedge e_{s}+\left(1-t_{j}\right) e_{r} \wedge e_{s}\right] & \text { if } r=i<j<s, \\
\left(t_{j}-1\right)\left[\left(1-t_{s}\right) e_{r} \wedge e_{i}+\left(1-t_{r}\right) e_{i} \wedge e_{s}\right] & \text { if } r<i<j<s, \\
\quad+\left(1-t_{i}\right)\left[\left(1-t_{s}\right) e_{r} \wedge e_{j}+\left(1-t_{r}\right) e_{j} \wedge e_{s}\right] & \\
0 & \text { otherwise. }\end{cases}
\end{aligned}
$$

If $\{r, s\} \not \subset V$, it is readily checked that $\pi_{U}\left(W_{i, j}^{\delta}\right) \in I_{U}^{\perp} \cdot C_{2}(U)$ for each $U \in L_{2}(\mathcal{A})$.

In general, write $\delta$ as the product of $A_{r, s}^{ \pm 1}$ and $\delta^{\prime}$, and assume inductively that $\Theta_{2}\left(\delta^{\prime}\right)\left(e_{i} \wedge e_{j}\right)=e_{i} \wedge e_{j}+W_{i, j}^{\prime}$ satisfies $\pi_{U}\left(W_{i, j}^{\prime}\right) \in I_{U}^{\perp} \cdot C_{2}(U)$ for each $U$. Then $\Theta_{2}(\delta)\left(e_{i} \wedge e_{j}\right)=\Theta_{2}\left(A_{r, s}^{ \pm 1}\right)\left(e_{i} \wedge e_{j}\right)+\Theta_{2}\left(A_{r, s}^{ \pm 1}\right)\left(W_{i, j}^{\prime}\right)$, and by the above, it remains to analyze the latter summand. If $w \cdot e_{p} \wedge e_{q}$ is a summand of $W_{i, j}^{\prime}$, then $w \in I_{V(p, q)}^{\perp}$ by induction. Case-by-case analysis then shows that each summand $x \cdot e_{\ell} \wedge e_{m}$ of $\Theta_{2}\left(A_{r, s}^{ \pm 1}\right)\left(w \cdot e_{p} \wedge e_{q}\right)$ satisfies $x \in I_{V(\ell, m)}^{\perp}$. This completes the proof of the claim, and hence that of the proposition.

We can now formulate the main result of this subsection.

Theorem 6.3. There exists a chain map $\Upsilon_{\bullet}$ from the presentation $K_{\bullet} \rightarrow B(\mathcal{A})$ to the resolution $L_{\bullet} \rightarrow B^{\mathrm{cc}}(\mathcal{A})$,

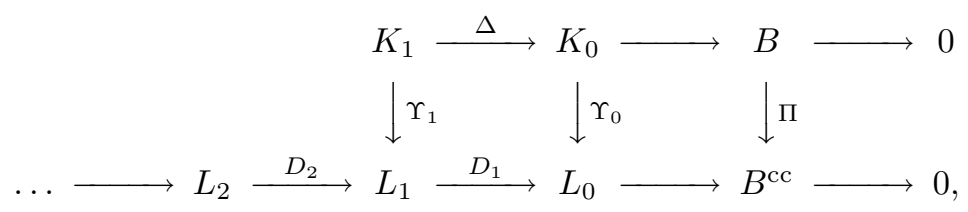

given by $\Upsilon_{0}=\Psi_{2}$, and $\Upsilon_{1}(x, y)=\Gamma(x)+\Psi_{3}(y)$. Furthermore, the resulting map $\Pi: B \rightarrow B^{\mathrm{cc}}$ is surjective.

Proof. It is immediate from the above that $\Upsilon_{\bullet}$ is a chain map. Thus it suffices to show that the map $\Upsilon_{0}: K_{0} \rightarrow L_{0}$, which by definition equals $\Psi_{2}=\sum_{V} \pi_{V^{\prime}} \circ$ $\Theta_{2}\left(\mu_{V}\right)^{-1}: C_{2} \rightarrow \bigoplus_{V} C_{2}\left(V^{\prime}\right)$, is surjective. With respect to the decomposition $K_{0}=K_{0}^{\prime} \oplus L_{0}=\left(\bigoplus_{V} C_{2}^{\prime}(V)\right) \oplus\left(\bigoplus_{V} C_{2}\left(V^{\prime}\right)\right)$, we have $\Upsilon_{0}=\left(\Upsilon_{0}^{\prime} \quad \operatorname{id}_{L_{0}}\right)^{\top}$ : $K_{0}^{\prime} \oplus L_{0} \rightarrow L_{0}$, where $\Upsilon_{0}^{\prime}\left(e_{i} \wedge x\right)=\left(e_{i}-\nabla_{V}\right) \wedge x$, for $i=\min V$ and $x \in C_{1}\left(V^{\prime}\right)$. Thus $\Upsilon_{0}: K_{0} \rightarrow L_{0}$ is surjective.

6.4. Decomposition of the Completion. Recall that if $B$ is a $\Lambda$-module, then $\widehat{B}$ denotes its $I$-adic completion, and that if $f: A \rightarrow B$ is a map of $\Lambda$-modules, we write $\widehat{f}: \widehat{A} \rightarrow \widehat{B}$ for the extension of $f$ to the completions. The $I$-adic completion functor takes chain complexes to chain complexes, and chain maps to chain maps. 
Theorem 6.5. The chain map $\widehat{\Upsilon}_{\bullet}: \widehat{K}_{\bullet} \rightarrow \widehat{L}_{\bullet}$ induces an isomorphism $\widehat{B} \stackrel{\sim}{\longrightarrow} \widehat{B}^{\text {cc }}$ if and only if the map $\widehat{\Psi}_{3}: \widehat{C}_{3} \rightarrow \widehat{L}_{1}$ is surjective.

Proof. Consider the mapping cone, $K_{\bullet}(\widehat{\Upsilon})$, of $\widehat{\Upsilon}_{\bullet}$, given by

$$
\cdots \rightarrow \widehat{L}_{2} \oplus \widehat{K}_{1} \stackrel{\partial_{2}}{\longrightarrow} \widehat{L}_{1} \oplus \widehat{K}_{0} \stackrel{\partial_{1}}{\longrightarrow} \widehat{L}_{0}
$$

where $\partial_{2}(x, y)=\left(\widehat{D}_{2}(x)-\widehat{\Upsilon}_{1}(y), \widehat{\Delta}(y)\right)$ and $\partial_{1}(x, y)=\widehat{D}_{1}(x)+\widehat{\Upsilon}_{0}(y)$, and the short exact sequence of chain complexes

$$
0 \rightarrow \widehat{L}_{\bullet} \stackrel{\bullet}{\longrightarrow} K_{\bullet}(\widehat{\Upsilon}) \stackrel{\pi_{\bullet}}{\longrightarrow} \widehat{K}_{\bullet-1} \rightarrow 0,
$$

where $\iota_{\bullet}$ and $\pi_{\bullet}$ denote the natural inclusion and projection. Since $\widehat{\Upsilon}_{0}$ is surjective, we have $H_{0}\left(K_{\bullet}(\widehat{\Upsilon})\right)=0$. Also, since $\widehat{L} \bullet$ is a resolution, $\widetilde{H}_{*}\left(\widehat{L}_{\bullet}\right)=0$. Thus the associated long exact sequence in homology reduces to

$$
0 \rightarrow H_{1}\left(K_{\bullet}(\widehat{\Upsilon})\right) \rightarrow H_{0}\left(\widehat{K}_{\bullet}\right) \stackrel{\widehat{\Upsilon}_{*}}{\longrightarrow} H_{0}\left(\widehat{L}_{\bullet}\right) \rightarrow 0 .
$$

The map $\widehat{\Upsilon}_{*}: H_{0}\left(\widehat{K}_{\bullet}\right)=H_{0}\left(\widehat{L}_{\bullet}\right)$ identifies canonically with $\widehat{\Pi}: \widehat{B} \rightarrow \widehat{B}^{\text {cc }}$. Thus, it suffices to show that $\operatorname{coker}\left(\widehat{\Psi}_{3}\right)=0$ if and only if $H_{1}\left(K_{\bullet}(\widehat{\Upsilon})\right)=0$.

Recall the map $\Phi: \bigoplus_{V} C_{1}\left(V^{\prime}\right) \rightarrow C_{2}$ from Theorem 5.3. Recall also (from the proof of Proposition 6.2) that $\left.\Phi\right|_{C_{1}\left(V^{\prime}\right)}=\Phi_{V} \bmod I$. Thus, $\left.\widehat{\Phi}\right|_{\widehat{C}_{1}\left(V^{\prime}\right)}=\widehat{\Phi}_{V} \bmod \mathfrak{m}$. Using the identification $C_{1}\left(V^{\prime}\right) \stackrel{\sim}{\longrightarrow} C_{2}^{\prime}(V), x \mapsto e_{i} \wedge x$, where $i=\min V$, and the projection on the first factor $p^{\prime}: K_{0}=K_{0}^{\prime} \oplus L_{0} \rightarrow K_{0}^{\prime}$, define $\Phi^{\prime}:=p^{\prime} \circ \Phi: K_{0}^{\prime} \rightarrow K_{0}^{\prime}$. Since $\widehat{\nabla}_{V}=\sum_{i \in V} e_{i} \bmod \mathfrak{m}$, the map $\widehat{p}^{\prime} \circ \widehat{\Phi}_{V}: \widehat{C}_{1}\left(V^{\prime}\right) \rightarrow \widehat{C}_{2}^{\prime}(V)$ coincides with the above identification. Hence, $\widehat{\Phi}^{\prime}=$ id $\bmod \mathfrak{m}$. Consequently, $\widehat{\Phi}^{\prime}$ is an isomorphism.

We now alter the short exact sequence (6.3). Write $K_{1}(\widehat{\Upsilon})=\widehat{L}_{1} \oplus \widehat{K}_{0}^{\prime} \oplus \widehat{L}_{0}$ and $K_{2}(\widehat{\Upsilon})=\widehat{L}_{2} \oplus \widehat{K}_{0}^{\prime} \oplus \widehat{C}_{3}$, and define $\rho \in \operatorname{Aut} K_{1}(\widehat{\Upsilon})$ and $\psi \in \operatorname{Aut} K_{2}(\widehat{\Upsilon})$ by

$$
\begin{aligned}
& \rho(x, y, z)=\left(x+\widehat{\Gamma} \circ \widehat{\Phi}^{\prime-1}(y), y, D_{1}(x)+\widehat{\Upsilon}_{0}^{\prime}(y)+z\right), \\
& \psi(x, y, z)=\left(x, \widehat{\Phi}^{\prime-1}(y)-\widehat{\Phi}^{\prime-1} \circ p^{\prime} \circ \widehat{d}_{3}(z), z\right) .
\end{aligned}
$$

Note that the restriction of $\psi$ to $\widehat{L}_{2}$ is the identity, and let $\bar{\psi}$ denote the restriction of $\psi$ to $\widehat{K}_{1}=\widehat{K}_{0}^{\prime} \oplus \widehat{C}_{3}$. We modify the sequence (6.3) as indicated below.

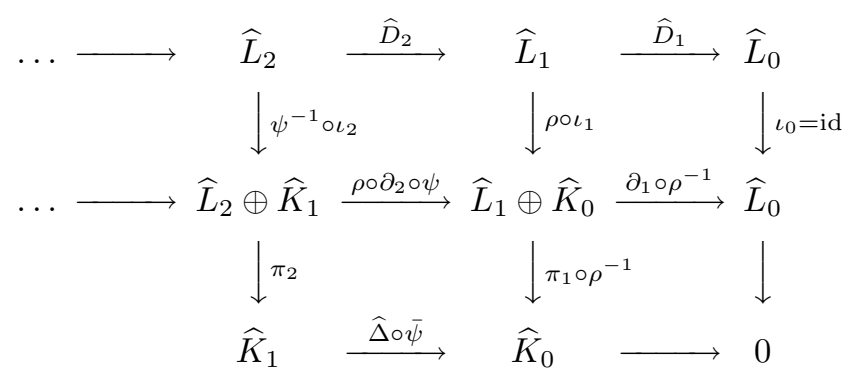

Since $\bar{\psi} \circ \pi_{2}=\pi_{2} \circ \psi$, this diagram commutes.

Consider the map $\Xi: \widehat{L}_{2} \oplus \widehat{C}_{3} \rightarrow \widehat{L}_{1}$ defined by

$$
\Xi(x, z)=\widehat{D}_{2}(x)+\widehat{\Gamma} \circ \widehat{\Phi}^{\prime-1} \circ p^{\prime} \circ \widehat{d}_{3}(z)-\widehat{\Psi}_{3}(z) .
$$

Computations with the definitions (making use of the fact that $\widehat{\Upsilon}_{\bullet}$ is a chain map) reveal that $\partial_{1} \circ \rho^{-1}(x, y, z)=z$ and $\rho \circ \partial_{2} \circ \psi(x, y, z)=(\Xi(x, z), y, 0)$. Thus, $\Xi$ provides a presentation for the module $H_{1}\left(K_{\bullet}(\widehat{\Upsilon})\right)$, and this module is trivial if and 
only if $\Xi$ is surjective. Since $\operatorname{im}\left(\widehat{D}_{2}\right) \subset \mathfrak{m} \cdot \widehat{L}_{1}$ and $\operatorname{im}\left(\widehat{\Gamma} \circ \widehat{\Phi}^{\prime-1} \circ p^{\prime} \circ \widehat{d}_{3}\right) \subset \mathfrak{m} \cdot \widehat{L}_{1}$, the map $\Xi$ is surjective if and only if $\widehat{\Psi}_{3}$ is surjective.

The above proof has several consequences, even in the instance when the map $\widehat{\Psi}_{3}$ is not surjective, see below and Theorem 7.5. These results hold for an arbitrary arrangement (real or complex, compare 5.3 and 5.5) that is transverse to infinity.

Corollary 6.6. The I-adic completion $\widehat{B}$ of the Alexander invariant, an arrangement $\mathcal{A}$, has a presentation with $\left(\begin{array}{l}n \\ 2\end{array}\right)-b_{2}(M(\mathcal{A}))$ generators, and $\left(\begin{array}{l}n \\ 3\end{array}\right)$ relations.

Proof. From the commutative diagram (6.5), we have the presentation $\widehat{\Delta} \circ \bar{\psi}: \widehat{K}_{1} \rightarrow$ $\widehat{K}_{0}$ for $\widehat{B}$. Let $p^{\prime \prime}: K_{0}=K_{0}^{\prime} \oplus L_{0} \rightarrow L_{0}$ denote the projection onto second factor. With respect to the decompositions $\widehat{K}_{1}=\widehat{K}_{0}^{\prime} \oplus \widehat{C}_{3}$ and $\widehat{K}_{0}=\widehat{K}_{0}^{\prime} \oplus \widehat{L}_{0}$, the map $\widehat{\Delta} \circ \bar{\psi}: \widehat{K}_{1} \rightarrow \widehat{K}_{0}$ is given by

$$
\widehat{\Delta} \circ \bar{\psi}(x, y)=\left(x, \widehat{p}^{\prime \prime}\left(\widehat{\Phi} \circ \widehat{\Phi}^{\prime-1}(x)-\widehat{\Phi} \circ \widehat{\Phi}^{\prime-1} \circ \widehat{p}^{\prime} \circ \widehat{d}_{3}(y)+\widehat{d}_{3}(y)\right)\right) .
$$

Define

$$
\widehat{\Delta}^{\sharp}=\widehat{p}^{\prime \prime} \circ\left(\mathrm{id}-\widehat{\Phi} \circ \widehat{\Phi}^{\prime-1} \circ \widehat{p}^{\prime}\right) \circ \widehat{d}_{3}: \widehat{C}_{3} \rightarrow \widehat{L}_{0},
$$

and define $\chi \in$ Aut $\widehat{K}_{0}$ by $\chi(x, y)=\left(x, y-\widehat{p}^{\prime \prime} \circ \widehat{\Phi} \circ \widehat{\Phi}^{\prime-1}(x)\right)$. Then $\chi \circ \widehat{\Delta} \circ \bar{\psi}(x, y)=$ $\left(x, \widehat{\Delta}^{\sharp}(y)\right)$. Thus, $\widehat{\Delta}^{\sharp}$ provides a presentation for $\widehat{B}$ with the specified numbers of generators and relations.

\section{Combinatorics and the Chen groups}

In this section, we examine the relationship between the results obtained in the previous sections and the combinatorics of a hyperplane arrangement. We refer to the Chen groups of the group $G$ of an arrangement $\mathcal{A}$ as simply the Chen groups of $\mathcal{A}$.

An invariant of $\mathcal{A}$ is called combinatorial if it is determined by the isomorphism type of the lattice $L(\mathcal{A})$. As is well-known from [12], the ranks $\phi_{k}$ of the LCS quotients of the group of $\mathcal{A}$ are combinatorially determined. Thus, the ranks of the first three Chen groups of $\mathcal{A}$ are combinatorial. We now describe some explicit combinatorial bounds and formulas for the ranks $\theta_{k}$ of the Chen groups of $\mathcal{A}$.

7.1. A Bound on Chen Ranks. Recall that the coarse combinatorial Alexander invariant $B^{\text {cc }}$ of $\mathcal{A}$ is the direct sum $\bigoplus_{V} B_{V}$ of the Alexander invariants of the vertex groups $G_{V}$, indexed by $V \in L_{2}(\mathcal{A})$, the rank two elements of $L(\mathcal{A})$. For $k \geq 2$, define the coarse combinatorial Chen ranks by

$$
\theta_{k}^{\mathrm{cc}}(\mathcal{A})=\sum_{V \in L_{2}(\mathcal{A})} \theta_{k}\left(G_{V}\right)
$$

From Theorem 1.3 and Theorem 6.3, we obtain the following.

Corollary 7.2. For $k \geq 2$, the ranks of the Chen groups of $\mathcal{A}$ are bounded below by the coarse combinatorial Chen ranks: $\theta_{k}(\mathcal{A}) \geq \theta_{k}^{\mathrm{cc}}(\mathcal{A})$.

To compute these lower bounds explicitly, recall that $G_{V} \cong F_{r-1} \times \mathbb{Z}^{n-r}$, where $r=|V|$. By Corollary 1.10, the ranks of the Chen groups of $G_{V}$ are given by 
$\theta_{k}\left(G_{V}\right)=(k-1)\left(\begin{array}{c}k+r-3 \\ k\end{array}\right)$ for $k \geq 2$. Let $c_{r}$ denote the number of elements of $L_{2}(\mathcal{A})$ of multiplicity $r$, and write $\left(\begin{array}{c}m \\ k\end{array}\right)=0$ if $m<k$. Then,

$$
\theta_{k}^{\mathrm{cc}}=\sum_{V \in L_{2}(\mathcal{A})}(k-1)\left(\begin{array}{c}
k+|V|-3 \\
k
\end{array}\right)=\sum_{r \geq 3} c_{r}(k-1)\left(\begin{array}{c}
k+r-3 \\
k
\end{array}\right),
$$

and so $\theta_{k}^{\text {cc }}$ is determined by (only) the multiplicities of the elements of $L_{2}(\mathcal{A})$.

Remark 7.3. The ranks of the lower central series quotients of the group $G$ of $\mathcal{A}$ satisfy analogous lower bounds: $\phi_{k}(\mathcal{A}) \geq \phi_{k}^{\mathrm{cc}}(\mathcal{A})=\sum_{V \in L_{2}(\mathcal{A})} \phi_{k}\left(G_{V}\right)$, see [13], Proposition 3.8 .

Remark 7.4. The lower bounds for the ranks of the Chen groups of $\mathcal{A}$ may be expressed in terms of the Möbius function $\mu: L(\mathcal{A}) \rightarrow \mathbb{Z}$ :

$$
\theta_{k}(\mathcal{A}) \geq \sum_{V \in L_{2}(\mathcal{A})}(k-1)\left(\begin{array}{c}
k+\mu(V)-2 \\
k
\end{array}\right)
$$

We now analyze the difference $\theta_{k}-\theta_{k}^{\text {cc }}$. Recall that $\theta_{k}=\operatorname{rank}\left(\mathfrak{m}^{k-2} \widehat{B} / \mathfrak{m}^{k-1} \widehat{B}\right)$. Checking that the image of the map $\widehat{\Delta}^{\sharp}: \widehat{C}_{3} \rightarrow \widehat{L}_{0}$ defined in (6.7) is contained in $\mathfrak{m} \cdot \widehat{L}_{0}$, we see that $\theta_{2}(\mathcal{A})=\operatorname{rank}(\widehat{B} / \mathfrak{m} \widehat{B})=\left(\begin{array}{l}n \\ 2\end{array}\right)-b_{2}(M(\mathcal{A}))$, and thus $\theta_{2}(\mathcal{A})=$ $\theta_{2}^{\text {cc }}(\mathcal{A})$.

Recall the mapping cone $K_{\bullet}(\widehat{\Upsilon})$ from $(6.2)$, and set $H=H_{1}\left(K_{\bullet}(\widehat{\Upsilon})\right)$.

Theorem 7.5. For $k \geq 3$, the rank of the $k^{\text {th }}$ Chen group of $\mathcal{A}$ is given by $\theta_{k}(\mathcal{A})=$ $\operatorname{rank}\left(\mathfrak{m}^{k-3} H / \mathfrak{m}^{k-2} H\right)+\theta_{k}^{\text {cc }}(\mathcal{A})$. In particular, $\theta_{3}(\mathcal{A})=\operatorname{rank}\left(\operatorname{coker} \widehat{\Psi}_{3}\right)+\theta_{3}^{\text {cc }}(\mathcal{A})$.

Proof. Consider the short exact sequence (6.4), rewritten as

$$
0 \rightarrow H \stackrel{\tau}{\rightarrow} \widehat{B} \stackrel{\widehat{\Pi}}{\longrightarrow} \widehat{B}^{\mathrm{cc}} \rightarrow 0 .
$$

Altering the commutative diagram (6.5) using the isomorphism $\chi \in$ Aut $\widehat{K}_{0}$ defined in the proof of Corollary 6.6, we see that $\tau$ is induced by $\chi \circ \pi_{1} \circ \rho^{-1}: \widehat{L}_{1} \oplus$ $\widehat{K}_{0}^{\prime} \oplus \widehat{L}_{0} \rightarrow \widehat{K}_{0},(x, y, z) \mapsto\left(y, z-\widehat{D}_{1}(x)\right)$. Thus the restriction of this map to $\operatorname{ker}\left(\partial_{1} \circ \rho^{-1}\right)=\widehat{L}_{1} \oplus \widehat{K}_{0}^{\prime}$ is given by $(x, y) \mapsto\left(y,-\widehat{D}_{1}(x)\right)$. Since $\widehat{B}=\operatorname{coker}(\chi \circ \widehat{\Delta} \circ \bar{\psi})$ and $\chi \circ \widehat{\Delta} \circ \bar{\psi}(y, 0)=(y, 0)$, the map $\tau: H \rightarrow \widehat{B}$ in homology is induced by $\widehat{D}_{1}: \widehat{L}_{1} \rightarrow \widehat{L}_{0}$. Since $\operatorname{im}\left(\widehat{D}_{1}(z)\right) \subset \mathfrak{m} \cdot \widehat{L}_{0}$, from the exact sequence (7.1) we have

$$
\operatorname{rank}\left(\mathfrak{m}^{\ell} \widehat{B} / \mathfrak{m}^{\ell-1} \widehat{B}\right)=\operatorname{rank}\left(\mathfrak{m}^{\ell-1} H / \mathfrak{m}^{\ell-2} H\right)+\operatorname{rank}\left(\mathfrak{m}^{\ell} \widehat{B}^{\mathrm{cc}} / \mathfrak{m}^{\ell-1} \widehat{B}^{\mathrm{cc}}\right) .
$$

It follows from Theorem 1.3 that the ranks of the Chen groups of $\mathcal{A}$ are as asserted.

In particular, the third Chen group of $\mathcal{A}$ has $\operatorname{rank} \theta_{3}(\mathcal{A})=\operatorname{rank}(H / \mathfrak{m} H)+\theta_{3}^{\text {cc }}(\mathcal{A})$. Recall the presentation, $\Xi: \widehat{L}_{2} \oplus \widehat{C}_{3} \rightarrow \widehat{L}_{1}$, for the module $H$ from (6.6). Using elementary row and column operations, we obtain a presentation $\Xi^{\prime}: \widehat{\Lambda}^{a} \rightarrow \widehat{\Lambda}^{b}$ from this with $b=\operatorname{rank} \widehat{L}_{1}-\operatorname{rank} \widehat{\Psi}_{3}$ generators. Checking that $\operatorname{im}\left(\Xi^{\prime}\right) \subset \mathfrak{m} \cdot \widehat{\Lambda}^{b}$, we get $\operatorname{rank}(H / \mathfrak{m} H)=\operatorname{rank}\left(\operatorname{coker} \widehat{\Psi}_{3}\right)$.

7.6. Decomposition is Combinatorial. Let $\hat{\epsilon}: \widehat{\Lambda} \rightarrow \mathbb{Z}$ be the augmentation map, which takes a power series to its constant coefficient. If $\widehat{F}=\widehat{\Lambda}^{p}$ is a free module, denote its image under $\hat{\epsilon}$ by $\bar{F}=\mathbb{Z}^{p}$, and if $\widehat{f}: \widehat{F} \rightarrow \widehat{F}^{\prime}$ is a $\widehat{\Lambda}$-linear map, denote its image by $\bar{f}: \bar{F} \rightarrow \bar{F}^{\prime}$.

Lemma 7.7. The rank of $\widehat{f}$ is equal to the rank of $\bar{f}$. 
Proof. Suppose the rank of $\bar{f}: \mathbb{Z}^{p} \rightarrow \mathbb{Z}^{q}$ is $r$. Then there are integral matrices $X \in \mathrm{GL}(p, \mathbb{Z})$ and $Y \in \operatorname{GL}(q, \mathbb{Z})$ so that $X \cdot \bar{f} \cdot Y=\left(\begin{array}{cc}I_{r} & 0 \\ 0 & 0\end{array}\right)$, where $I_{r}$ denotes the $r \times r$ identity matrix. By definition, $\widehat{f}=\bar{f}+Z$, where the entries of $Z$ are in $\mathfrak{m}$. Thus, $X \cdot \widehat{f} \cdot Y=X \cdot \bar{f} \cdot Y+X \cdot Z \cdot Y$. Clearly, the rank of $X \cdot \widehat{f} \cdot Y$, and hence that of $\widehat{f}$, is $r$.

The converse follows from the functoriality of the construction.

We now show that the rank of the map $\widehat{\Psi}_{3}: \widehat{C}_{3} \rightarrow \widehat{L}_{1}$ is combinatorially determined. Thus, the criterion for decomposition of the $I$-adic completion of the Alexander invariant of Theorem 6.5 - the surjectivity of $\widehat{\Psi}_{3}$-is combinatorial as well. By the lemma, it suffices to show that the rank of $\bar{\Psi}_{3}: \bar{C}_{3} \rightarrow \bar{L}_{1}$ is combinatorially determined.

For this, let $\mathcal{A}$ and $\mathcal{A}^{*}$ be lattice-isomorphic arrangements of $n$ lines in $\mathbb{C}^{2}$ (which are transverse to the line at infinity). Let $\mathcal{W}$ be a braided wiring diagram associated to $\mathcal{A}$, and let $\bar{\Psi}_{3}: \bar{C}_{3} \rightarrow \bar{L}_{1}=\bigoplus_{V} \bar{C}_{2}\left(V^{\prime}\right) \wedge \bar{C}_{1}$ be the map defined by the vertex sets $\left\{V_{1}, \ldots, V_{s}\right\}$ of $\mathcal{W}$. Choose arbitrary orderings of the hyperplanes and rank two lattice elements of $\mathcal{A}^{*}$, and denote the elements of $L_{2}\left(\mathcal{A}^{*}\right)$ by $\left\{U_{1}, \ldots, U_{s}\right\}$. Then formally construct the map $\bar{\Psi}_{3}^{*}=\sum_{U} \bar{\Psi}_{U, 3}: \bar{C}_{3} \rightarrow \bar{L}_{1}^{*}=\bigoplus_{U} \bar{C}_{2}\left(U^{\prime}\right) \wedge \bar{C}_{1}$ using (4.10), the Magnus embedding, and the augmentation map $\hat{\epsilon}$.

Since $\mathcal{A}$ and $\mathcal{A}^{*}$ are lattice-isomorphic, there are permutations $\omega \in \Sigma_{n}$ and $\nu \in \Sigma_{s}$ so that $\omega\left(V_{k}\right)=U_{\nu(k)}$ for each $k, 1 \leq k \leq s$. The permutation $\omega$ induces an isomorphism $\omega_{k}: C_{k} \rightarrow C_{k}$ defined by $\omega_{k}\left(e_{J}\right)=e_{\omega(J)}$. The map $\bar{\Psi}_{3}$ is combinatorially determined in the sense of the following.

Proposition 7.8. There is an isomorphism $\bar{\xi}: \bar{L}_{1} \rightarrow \bar{L}_{1}^{*}$ so that $\bar{\xi} \circ \bar{\Psi}_{3}=\bar{\Psi}_{3}^{*} \circ \bar{\omega}_{3}$.

Proof. Let $V$ be a vertex set of $\mathcal{A}$, and $U=\omega(V)$ be the corresponding vertex set of $\mathcal{A}^{*}$. Define a map $\xi_{V}^{U}: C_{2}\left(V^{\prime}\right) \wedge C_{1} \rightarrow C_{2}\left(U^{\prime}\right) \wedge C_{1}$ by

$$
\xi_{V}^{U}=\left(\pi_{U^{\prime}} \wedge \mathrm{id}\right) \circ \Theta_{2}\left(\mu_{U}\right)^{-1} \circ \omega_{3} \circ \Theta_{2}\left(\mu_{V}\right) \circ\left(\iota_{V^{\prime}} \wedge \mathrm{id}\right) .
$$

Clearly, $\xi_{V}^{U}$ is an isomorphism, with inverse $\xi_{U}^{V}$. Moreover, $\xi_{V}^{U} \circ \Psi_{V, 3}=\Psi_{U, 3} \circ \omega_{3}$. The collection $\left\{\xi_{U}^{V}\right\}$ defines a map $\xi: L_{1} \rightarrow L_{1}^{*}$, which yields the desired isomorphism $\bar{\xi}$.

Combining these results with those of the previous section, we obtain

Theorem 7.9. The rank of the third Chen group of the arrangement $\mathcal{A}$ is given by the combinatorial formula $\theta_{3}(\mathcal{A})=\operatorname{rank}\left(\operatorname{coker} \bar{\Psi}_{3}\right)+\theta_{3}^{\mathrm{cc}}(\mathcal{A})$.

Furthermore, if the map $\bar{\Psi}_{3}: \bar{C}_{3} \rightarrow \bar{L}_{1}$ is surjective, then the I-adic completion of the Alexander invariant of $\mathcal{A}$ decomposes as a direct sum: $\widehat{B} \cong \widehat{B}^{\mathrm{cc}}=\bigoplus_{V} \widehat{B}_{V}$, and the ranks of the Chen groups of $\mathcal{A}$ are given by $\theta_{k}(\mathcal{A})=\theta_{k}^{\mathrm{cc}}(\mathcal{A})$ for all $k \geq 2$.

Remark 7.10. If $\mathcal{A}$ is an arrangement for which the map $\bar{\Psi}_{3}: \bar{C}_{3} \rightarrow \bar{L}_{1}$ is not surjective, the ranks $\theta_{k}(\mathcal{A})$ of the Chen groups of $\mathcal{A}$ for $k \geq 4$ may be computed using the Groebner basis algorithm described in 1.2. Alternatively, in light of Theorem 7.5, one can apply this algorithm to the presentation (6.6) of the module $H$ (or the smaller presentation described in the proof of Theorem 7.5) to determine $\operatorname{rank}\left(\mathfrak{m}^{k-3} H / \mathfrak{m}^{k-2} H\right)=\theta_{k}(\mathcal{A})-\theta_{k}^{\mathrm{cc}}(\mathcal{A})$. 


\section{EXAMPLES}

In this section, we illustrate the results of the previous sections by means of several explicit examples. We order the hyperplanes of an arrangement $\mathcal{A}=$ $\left\{H_{1}, \ldots, H_{n}\right\}$ in the order indicated by the defining polynomial $Q(\mathcal{A})=\prod_{k=1}^{n} \ell_{k}$ (so $H_{k}=\operatorname{ker} \ell_{k}$ ).

Example 8.1. Consider the central 3-arrangement $\mathcal{A}$ with defining polynomial $Q=x y z(y+z)(x-z)(2 x+y)$. Randell [28] noted that this arrangement is not $K(\pi, 1)$, and that there is no aspherical arrangement with the same lattice in ranks one and two. Arvola [1] further showed that the group of this arrangement is not of type FL.

The rank two elements of the lattice of $\mathcal{A}$ are

$$
\{\{1,2,6\},\{1,3,5\},\{2,3,4\},\{1,4\},\{2,5\},\{4,5\},\{3,6\},\{4,6\},\{5,6\}\} .
$$

It is readily checked that the map $\bar{\Psi}_{3}: \mathbb{Z}^{20} \rightarrow \mathbb{Z}^{12}$ is surjective. By Theorem 7.9 , $\widehat{B} \cong \widehat{B}^{\text {cc }}=\widehat{B}_{\{1,2,6\}} \oplus \widehat{B}_{\{1,3,5\}} \oplus \widehat{B}_{\{2,3,4\}}$. It follows that the ranks of the Chen groups of $\mathcal{A}$ are $\theta_{1}=6$ and $\theta_{k}=3(k-1)$ for $k \geq 2$. Notice that these ranks coincide with those of the Chen groups of a direct product of three free groups on two generators, though clearly $G \neq F_{2} \times F_{2} \times F_{2}$.

Using Theorem 5.5 and elementary row operations, one can show that the Alexander invariant itself decomposes as a direct sum, $B \cong B^{\text {cc }}=B_{\{1,2,6\}} \oplus$ $B_{\{1,3,5\}} \oplus B_{\{2,3,4\}}$.

Example 8.2. The braid arrangement $\mathcal{A}_{4}$ is the smallest arrangement for which the completion of the Alexander invariant does not decompose. The polynomial $Q=x y z(x-y)(x-z)(y-z)$ defines a central 3-arrangement whose complement is homotopy equivalent to that of $\mathcal{A}_{4}$. The rank two elements of $L\left(\mathcal{A}_{4}\right)$ (the partition lattice) are $\{\{1,2,4\},\{1,3,5\},\{2,3,6\},\{3,4\},\{2,5\},\{4,5,6\},\{1,6\}\}$. The map $\bar{\Psi}_{3}$ : $\mathbb{Z}^{20} \rightarrow \mathbb{Z}^{16}$ is not surjective. Thus $\widehat{B}\left(\mathcal{A}_{4}\right)$ does not decompose. A basis for coker $\bar{\Psi}_{3}$ is given by the two elements

$$
\begin{aligned}
& \kappa_{1}=e_{\{2,4\}} \wedge\left(e_{6}-e_{3}\right)+e_{\{3,5\}} \wedge\left(e_{4}-e_{6}\right)+e_{\{3,6\}} \wedge\left(e_{1}-e_{4}\right)+e_{\{5,6\}} \wedge\left(e_{3}-e_{1}\right), \\
& \kappa_{2}=e_{\{2,4\}} \wedge\left(e_{6}-e_{5}\right)+e_{\{3,5\}} \wedge\left(e_{2}-e_{6}\right)+e_{\{3,6\}} \wedge\left(e_{1}-e_{5}\right)+e_{\{5,6\}} \wedge\left(e_{2}-e_{1}\right) .
\end{aligned}
$$

Since $\operatorname{rank} \bar{\Psi}_{3}=14$, we have $\theta_{3}\left(\mathcal{A}_{4}\right)=10$.

Remark 8.3. Note that the rank of the third Chen group of $\mathcal{A}_{4}$ is equal to that of the product arrangement defined by $x y(y-x) z(z-x)(z-2 x)$. In general, by the LCS formula [15], the ranks of the lower central series quotients of the pure braid group $P_{n}$ are equal to those of the direct product of free groups $\Pi_{n}=F_{n-1} \times \cdots \times F_{1}$. These groups are distinguished by their Chen groups. For $k \geq 4$, we have $\theta_{k}\left(\Pi_{n}\right)=$ $(k-1)\left(\begin{array}{c}n+k-2 \\ k+1\end{array}\right)$, by Example 1.11, and $\theta_{k}\left(P_{n}\right)=(k-1)\left(\begin{array}{c}n+1 \\ 4\end{array}\right)$, by the main result of [5]. Thus, $\theta_{k}\left(P_{n}\right) \neq \theta_{k}\left(\Pi_{n}\right)$ for $n \geq 4$, and the groups $P_{n}$ and $\Pi_{n}$ are not isomorphic.

Remark 8.4. Example 8.2 provides an easy means for detecting when the completion of the Alexander invariant of an arrangement $\mathcal{A}$ does not decompose. If $\mathcal{S} \subset \mathcal{A}$ is a subarrangement which is lattice-isomorphic to the braid arrangement $\mathcal{A}_{4}$, one can use the above elements of coker $\bar{\Psi}_{3}\left(\mathcal{A}_{4}\right)$ and maps of the form (7.2) to generate non-trivial elements of coker $\bar{\Psi}_{3}(\mathcal{A})$. 
It is interesting to note that (the matroid of) such an arrangement $\mathcal{A}$ has "nonlocal decomposable relations," see [14].

Example 8.5 (Diamond). Let $\mathcal{D}$ be the central 3-arrangement with defining polynomial $Q(\mathcal{D})=x(x+y+z)(x+y-z) y(x-y-z)(x-y+z) z$. This is a free, simplicial arrangement for which the LCS formula does not hold, and the OrlikSolomon algebra is not quadratic, see [12], [16], [30]. The rank two elements of $L(\mathcal{D})$ are

$$
\{\{3,4,5\},\{1,2,5\},\{1,4\},\{1,3,6\},\{2,4,6\},\{1,7\},\{2,3,7\},\{4,7\},\{5,6,7\}\} .
$$

This arrangement has three distinct subarrangements lattice-isomorphic to $\mathcal{A}_{4}$. One such subarrangement is $\mathcal{S}=\mathcal{D} \backslash\left\{H_{1}\right\}$. Define $\omega:[6] \hookrightarrow[7]$ by $1 \mapsto 2,2 \mapsto 3$, $3 \mapsto 4,4 \mapsto 7,5 \mapsto 6,6 \mapsto 5$. The map $\omega$ gives rise to a lattice-isomorphism $L\left(\mathcal{A}_{4}\right) \stackrel{\sim}{\longrightarrow} L(\mathcal{S})$. Define $\bar{\xi}: \bigoplus_{V \in L_{2}\left(\mathcal{A}_{4}\right)} \bar{C}_{2}\left(V^{\prime}\right) \wedge \bar{C}_{1} \rightarrow \bigoplus_{U \in L_{2}(\mathcal{D})} \bar{C}_{2}\left(U^{\prime}\right) \wedge \bar{C}_{1}$ as in (7.2): $\bar{\xi}\left(e_{\{5,6\}} \wedge e_{j}\right)=-e_{\{6,7\}} \wedge \bar{\omega}_{1}\left(e_{j}\right)$ and $\bar{\xi}\left(e_{K} \wedge e_{j}\right)=\bar{\omega}_{3}\left(e_{K} \wedge e_{j}\right)$ for $K \neq\{5,6\}$. Recall the elements $\kappa_{1}$ and $\kappa_{2}$ from Example 8.2, and let $\kappa_{i}^{\prime}=\bar{\xi}\left(\kappa_{i}\right)$. Then

$\kappa_{1}^{\prime}=e_{\{3,7\}} \wedge\left(e_{5}-e_{4}\right)+e_{\{4,6\}} \wedge\left(e_{7}-e_{5}\right)+e_{\{4,5\}} \wedge\left(e_{2}-e_{7}\right)+e_{\{6,7\}} \wedge\left(e_{2}-e_{4}\right)$, $\kappa_{2}^{\prime}=e_{\{3,7\}} \wedge\left(e_{5}-e_{6}\right)+e_{\{4,6\}} \wedge\left(e_{3}-e_{5}\right)+e_{\{4,5\}} \wedge\left(e_{2}-e_{6}\right)+e_{\{6,7\}} \wedge\left(e_{2}-e_{3}\right)$

are in the cokernel of $\bar{\Psi}_{3}: \mathbb{Z}^{35} \rightarrow \mathbb{Z}^{30}$. We obtain 6 distinct elements of coker $\bar{\Psi}_{3}$ in this way. However, there is a relation among them. We have $\operatorname{rank} \bar{\Psi}_{3}=25$, and $\theta_{3}(\mathcal{D})=17$.

The ranks of the higher Chen groups may be found via the Groebner basis algorithm of Theorem 1.5. By Example 1.12, we can simplify the computation by working with the decone of $\mathcal{D}$ defined by $\left.Q(\mathcal{D})\right|_{z=1}$. Rotating this arrangement counterclockwise to insure that first coordinate projection is generic, we obtain a wiring diagram $\mathcal{W}=\{\{3,4,5\},\{1,2,5\},\{1,4\},\{1,3,6\},\{2,4,6\}\}$. The image of the associated braid monodromy $\alpha: F_{5} \rightarrow P_{6}$ is generated by $\left\{A_{3,4,5}, A_{1,2,5}\right.$, $\left.A_{1,4}^{A_{3,4}}, A_{1,3,6}, A_{2,4,6}^{A_{3,4} A_{3,6}}\right\}$. From the presentation $\Lambda^{20} \stackrel{\Delta}{\longrightarrow} \Lambda^{6} \rightarrow B \rightarrow 0$ provided by Theorem 5.5, we find $\theta_{k}(\mathcal{D})=9(k-1)$, for $k \geq 4$.

We have found a number of other arrangements for which the completion of the Alexander invariant does not decompose as a direct sum. For example, for the Coxeter arrangement of type $\mathrm{B}_{3}$, we have $\bar{C}_{3}=\mathbb{Z}^{84}, \bar{L}_{1}=\mathbb{Z}^{85}$, and $\bar{\Psi}_{3}: \bar{C}_{3} \rightarrow \bar{L}_{1}$ is obviously not surjective. (This arrangement also has (many) subarrangements lattice-isomorphic to $\mathcal{A}_{4}$.) More subtle examples include the following.

Example 8.6 (MacLane). The polynomials

$$
Q^{ \pm}=x y(y-x) z\left(z-x-\omega^{2} y\right)(z+\omega y)(z-x)\left(z+\omega^{2} x+\omega y\right)
$$

where $\omega=(-1 \pm \sqrt{-3}) / 2$, define complex conjugate realizations $\mathcal{A}^{ \pm}$of the MacLane matroid (the $8_{3}$ configuration). These arrangements were used by Rybnikov [29] in his construction of lattice-isomorphic arrangements with distinct fundamental groups. Rybnikov's arrangements are not distinguished by their Chen groups.

Complex conjugation induces an isomorphism of the groups of $\mathcal{A}^{+}$and $\mathcal{A}^{-}$, and thus an isomorphism of the Alexander invariants, $B^{+} \cong B^{-}$. Neither of these arrangements has a subarrangement lattice-isomorphic to the braid arrangement $\mathcal{A}_{4}$. Nevertheless, the maps $\bar{\Psi}_{3}^{ \pm}$are not surjective, and the modules $\widehat{B}^{ \pm}$do not decompose. 
The hyperplane $H=\{7 x-5 y+z=1\}$ is generic with respect to both these arrangements. Moreover, the projection pr: $\mathbb{C}^{2} \rightarrow \mathbb{C}$ defined by $\operatorname{pr}(x, y)=13 x-4 y$ is generic with respect to both affine 2-arrangements $H \cap \mathcal{A}^{ \pm}$. Changing coordinates accordingly, we obtain braided wiring diagrams $\mathcal{W}^{ \pm}=\left\{V_{1}, \beta_{1}^{ \pm}, V_{2}, \beta_{2}^{ \pm}, \ldots, \beta_{11}^{ \pm}, V_{12}\right\}$ with vertex sets and intermediary braids given by:

$$
\begin{array}{llll}
V_{1}=\{3,4\}, & \beta_{1}^{ \pm}=1, & V_{7}=\{2,5,7\}, & \beta_{7}^{ \pm}=\sigma_{3}^{\mp 1}, \\
V_{2}=\{3,5,6\}, & \beta_{2}^{ \pm}=1, & V_{8}=\{6,7\}, & \beta_{8}^{ \pm}=\sigma_{4}^{ \pm 1} \sigma_{3}^{ \pm 1}, \\
V_{3}=\{3,7,8\}, \quad \beta_{3}^{ \pm}=1, & V_{9}=\{1,6,8\}, & \beta_{9}^{ \pm}=\sigma_{1}^{\mp 1}, \\
V_{4}=\{2,4,6\}, & \beta_{4}^{ \pm}=\sigma_{4}^{\mp 1} \sigma_{3}^{\mp 1}, & V_{10}=\{1,4,7\}, & \beta_{10}^{ \pm}=\sigma_{2}^{\mp 1}, \\
V_{5}=\{2,8\}, & \beta_{5}^{ \pm}=1, & V_{11}=\{1,5\}, & \beta_{11}^{ \pm}=\sigma_{4}^{ \pm 1}, \\
V_{6}=\{4,5,8\}, & \beta_{6}^{ \pm}=\sigma_{2}^{ \pm 1} \sigma_{5}^{ \pm 1}, & V_{12}=\{1,2,3\} . &
\end{array}
$$

An argument as in [7] shows that the braid monodromies associated to $\mathcal{W}^{+}$and $\mathcal{W}^{-}$are equivalent, but not braid equivalent. Calculations with these monodromies reveal that $\theta_{3}^{\text {cc }}\left(\mathcal{A}^{ \pm}\right)=16, \theta_{3}\left(\mathcal{A}^{ \pm}\right)=21$, and $\theta_{k}\left(\mathcal{A}^{ \pm}\right)=\theta_{k}^{\text {cc }}\left(\mathcal{A}^{ \pm}\right)=8(k-1)$ for $k \geq 4$. Thus the failure of $\bar{\Psi}_{3}$ to be surjective is detected only by the third Chen group.

Example 8.7 ( $9_{3}$ Configurations). The relationship between the (completion of the) Alexander invariant and the combinatorics of an arrangement appears to be quite delicate. As an illustration, consider the arrangements $\mathcal{P}_{1}$ and $\mathcal{P}_{2}$ defined by

$$
\begin{aligned}
& Q\left(\mathcal{P}_{1}\right)=x y z(x-y)(y-z)(x-y-z)(2 x+y+z)(2 x+y-z)(2 x-5 y+z), \\
& Q\left(\mathcal{P}_{2}\right)=x y z(x+y)(y+z)(x+3 z)(x+2 y+z)(x+2 y+3 z)(2 x+3 y+3 z) .
\end{aligned}
$$

The arrangement $\mathcal{P}_{1}$ is a realization of the Pappus configuration $\left(9_{3}\right)_{1}$, while $\mathcal{P}_{2}$ is a realization of the configuration $\left(9_{3}\right)_{2}$. Note that neither of these arrangements has a subarrangement lattice-isomorphic to the braid arrangement.

The combinatorial distinction between these arrangements (resp., their underlying matroids) is detected by the maps $\bar{\Psi}_{3}\left(\mathcal{P}_{k}\right): \mathbb{Z}^{84} \rightarrow \mathbb{Z}^{63}$. The map $\bar{\Psi}_{3}\left(\mathcal{P}_{2}\right)$ is surjective, and consequently the module $\widehat{B}\left(\mathcal{P}_{2}\right)$ decomposes as a direct sum. Thus, $\theta_{k}\left(\mathcal{P}_{2}\right)=9(k-1)$ for $k \geq 2$. However, the map $\bar{\Psi}_{3}\left(\mathcal{P}_{1}\right)$ is not surjective, and $\widehat{B}\left(\mathcal{P}_{1}\right)$ does not decompose. A calculation shows that $\theta_{2}\left(\mathcal{P}_{1}\right)=9$, and $\theta_{k}\left(\mathcal{P}_{1}\right)=10(k-1)$ for $k \geq 3$.

It was conjectured in [5] that, for $k$ sufficiently large, one has $\theta_{k}(\mathcal{A})=\theta_{k}^{\text {cc }}(\mathcal{A})+$ $(k-1) \beta(\mathcal{A})$, where $\beta(\mathcal{A})$ is the number of subarrangements of $\mathcal{A}$ that are latticeisomorphic to $\mathcal{A}_{4}$. The arrangement $\mathcal{P}_{1}$ has $\beta=0$ and $\theta_{k}^{\text {cc }}=9(k-1)$, and hence provides a counterexample to that conjecture.

\section{REFERENCES}

[1] W. Arvola, Arrangements and cohomology of groups, preprint.

[2] T. Becker, V. Weispfenning, Gröbner bases, Grad. Texts in Math., vol. 141, Springer-Verlag, New York-Berlin-Heidelberg, 1993. MR 95e:13018

[3] J. Birman, Braids, links and mapping class groups, Annals of Math. Studies, vol. 82, Princeton Univ. Press, Princeton, NJ, 1975. MR 51:11477

[4] K. T. Chen, Integration in free groups, Annals of Math. 54 (1951), 147-162. MR 13:105c

[5] D. Cohen, A. Suciu, The Chen groups of the pure braid group, In: Proceedings of the Cech Centennial Homotopy Theory Conference, Contemp. Math., vol. 181, Amer. Math. Soc., Providence, RI, 1995, pp. 45-64. MR 96c:20055

[6] _ Homology of iterated semidirect products of free groups, J. Pure Appl. Algebra 126 (1998), 87-120. CMP 98:07

[7] _ The braid monodromy of plane algebraic curves and hyperplane arrangements, Comment. Math. Helvetici 72 (1997), 285-315. MR 98f:52012 
[8] R. Cordovil, J. Fachada, Braid monodromy groups of wiring diagrams, Boll. Unione Mat. Ital. 9 (1995), 399-416. MR 96e:20057

[9] D. Cox, J. Little, D. O'Shea, Ideals, varieties, and algorithms, 2nd ed., Undergrad. Texts Math., Springer-Verlag, New York-Berlin-Heidelberg, 1997. MR 93j:13031

[10] R. H. Crowell, Torsion in link modules, J. Math. Mech. 14 (1965), 289-298. MR 30:4807

[11] 43:2055

[12] M. Falk, The minimal model of the complement of an arrangement of hyperplanes, Trans. Amer. Math. Soc. 309 (1988), 543-556. MR 89d:32024

[13] _ The cohomology and fundamental group of a hyperplane complement, In: Singularities, Contemp. Math., vol. 90, Amer. Math. Soc., Providence, RI, 1989, pp. 55-72. MR 90h:32026

[14] _ Arrangements and cohomology, Ann. Combin. 1 (1997), 135-157. CMP 98:14

[15] M. Falk, R. Randell, The lower central series of a fiber-type arrangement, Invent. Math. 82 (1985), 77-88. MR 87c:32015b

[16] On the homotopy theory of arrangements, In: Complex Analytic Singularities, Adv. Stud. Pure Math., vol. 8, North Holland, Amsterdam, 1987, pp. 101-124. MR 88f:32045

[17] R. Fox, Free differential calculus I, Annals of Math. 57 (1953), 547-560; II, 59 (1954), 196210; III, 64 (1956), 407-419. MR 14:843d; MR 15:931e; MR 20:2374

[18] M. Goresky, R. MacPherson, Stratified Morse theory, Ergeb. Math. Grenzgeb., vol. 14, Springer-Verlag, New York-Berlin-Heidelberg, 1988. MR 84k:58017

[19] H. Hamm, Lê D. T., Un théorème de Zariski du type de Lefschetz, Ann. Sci. École Norm. Sup. 6 (1973), 317-366. MR 53:5582

[20] E. Hironaka, Alexander stratifications of character varieties, Ann. Inst. Fourier (Grenoble) 47 (1997), 555-583. MR 98e:14020

[21] A. Libgober, On the homotopy type of the complement to plane algebraic curves, J. Reine Angew. Math. 367 (1986), 103-114. MR 87j:14044

[22] _ Abelian branched covers of projective plane, In: Singularity Theory, London Mathematical Society Lecture Note Series (J. W. Bruce, D. Mond, eds.), Cambridge Univ. Press, 1999, to appear.

[23] W. Massey, Completion of link modules, Duke Math. J. 47 (1980), 399-420. MR 81g:57004

[24] W. Massey, L. Traldi, On a conjecture of K. Murasugi, Pacific J. Math. 124 (1986), 193-213. MR 87k:57008

[25] S. Moran, The mathematical theory of knots and braids, North Holland Math. Stud., vol. 82, North Holland, Amsterdam, 1983. MR 85i:57001

[26] K. Murasugi, On Milnor's invariants for links. II. The Chen groups, Trans. Amer. Math. Soc. 148 (1970), 41-61. MR 41:4519

[27] P. Orlik, H. Terao, Arrangements of hyperplanes, Grundlehren Math. Wiss., vol. 300, Springer-Verlag, New York-Berlin-Heidelberg, 1992. MR 94e:52014

[28] R. Randell, Homotopy and group cohomology of arrangements, Top. and Appl. 20 (1996), 1-13. MR 98f:52014

[29] G. Rybnikov, On the fundamental group of the complement of a complex hyperplane arrangement, DIMACS Tech. Report 94-13 (1994), pp. 33-50; math. AG/9805056.

[30] B. Shelton, S. Yuzvinsky, Koszul algebras from graphs and hyperplane arrangements, J. London Math. Soc. 56 (1997), 477-490. CMP 98:09

[31] L. Traldi, The determinantal ideals of link modules. I, Pacific J. Math. 101 (1982), 215-222. MR 84h:57004

[32] O. Zariski, P. Samuel, Commutative algebra, vols. 1 and 2, reprint of the 1958-1960 edition, Springer-Verlag, New York-Berlin-Heidelberg, 1979. MR 52:5641; MR 52:10706

Department of Mathematics, Louisiana State University, Baton Rouge, Louisiana 70803

E-mail address: cohen@math.lsu.edu

URL: http://math.1su.edu/ ${ }^{\sim}$ cohen

Department of Mathematics, Northeastern University, Boston, Massachusetts 02115

E-mail address: alexsuciu@neu.edu

$U R L:$ http://www.math.neu.edu/ suciu 